\title{
An Integrated Genetic Map for Brassica napus Derived from Double Haploid and Recombinant Inbred Populations
}

\author{
Jianfeng Geng, Nasir Javed, Peter B.E. McVetty, Genyi Li and Muhammad Tahir*
}

Department of Plant Science, University of Manitoba, 222 Agriculture Building, 66 Dafoe Road, Winnipeg Manitoba, Canada

\begin{abstract}
A hybrid developed from a cross between two diverse Brassica napus cultivars ("Polo" and "Topas") was used to produce a microspore derived double haploid (DH) population and a single seed descent derived recombinant inbred (RI) population for genetic mapping. Each of the two populations consisting of $190 \mathrm{DH}$ lines and $94 \mathrm{RI}$ lines was characterized for various types (SSR, SRAP, ISSR, SCAR) of polymorphic molecular markers. The DH population was scored for 620 molecular markers while the RI population was scored for 349 molecular markers to construct two independent genetic maps. In both genetic maps, all of the molecular markers were found to cluster in 19 linkage groups (LGs) covered a total genome length of $2244.1 \mathrm{cM}$ and $1649.1 \mathrm{cM}$ for the DH and RI maps, respectively. The data from the two genetic maps was used to construct a consensus integrated genetic map covering a total genome length of $2464.9 \mathrm{cM}$. Previously published Brassica reference genetic maps were used to assign each of the nineteen LGs to corresponding Brassica napus chromosomes named N01 to N19. To our knowledge, this is the first integrated genetic map based on DH and RI populations developed from the same cross in Brassica napus.
\end{abstract}

Keywords: Brassica napus; Integrated genetic map

\section{Introduction}

Genetic maps of crop plants are now considered standard tools or even "road maps" [1], not only to understand genome structure and organization but also to tag economically important traits or genes. Such maps are developed by following the inheritance of detectable markers or genes in segregating populations derived from crosses of diverse parents. Rapid development in the field of molecular biology has allowed the use of molecular markers for the construction of high density genetic maps by exploiting variations (polymorphism) at the DNA level. Since the first use of restriction fragment length polymorphism (RFLP) as molecular markers followed by several other types of first generation markers, more than 30 types of $2^{\text {nd }}$ and $3^{\text {rd }}$ generation molecular markers are now used for the construction of genetic maps [2]. Among different types of $2^{\text {nd }}$ generation molecular markers, SSRs (simple sequence repeats) are becoming the preferred markers of choice for construction of genetic maps, tagging genes and assessing genetic diversity. This is largely due to the many useful features of SSRs such as co-dominant inheritance, multi-allelic nature with high polymorphism, abundance and even distribution in genomes, the low amount of DNA required for their detection by Polymerase Chain Reaction (PCR) and their suitablity for high-throughput analysis [3]. The SSRs are also ideal for anchoring molecular linkage maps since they are readily transferable among mapping populations [4]. This unique feature has been exploited to anchor genetic maps to physical maps in many important crop plants such as barley [5], cotton [6], Brassica rapa [7], and melon [8]. Sequence-related amplified polymorphism (SRAP), intersimple sequence repeat (ISSR) and sequence-characterized amplified region (SCAR) are some of the new generation markers which are getting increased usage in the construction of genetic maps due to various desirable features [9-15].

Availability of various types of useful molecular markers as described above and several efficient methods now available for the development of segregating populations (Double Haploid, Recombinant Inbreds etc.) has provided opportunities to construct integrated genetic maps using multiple types of segregating populations and molecular markers. This approach is becoming popular in map construction since a large number of potentially useful markers can be mapped and validated in various genetic backgrounds. Consquently, greater genome coverage is obtained [16]. A number of integrated genetic maps using multiple segregating populations and multiple types of molecular markers were constructed in sorghum [4,17-21], red clover pepper soybean ryegrass and common bean.

Brassica napus is the second most important oilseed crop in the world after soybean [22], and there is tremendous interest to understand the genetic structure and genome organization of this plant species including the construction of genetic maps. Many genetic maps of Brassica species have been published in recent years which are mainly based on a single type of population [23-29]. A number of studies [7,10,16,30-35], have also reported genetic maps based on multiple molecular markers and population types.

In this study we have attempted to construct genetic maps of Brassica napus using two types of mapping populations (a DH and a RI) segregating for various types of molecular markers. These two maps were further combined into an integrated genetic map. The genetic maps are mainly populated with previously published SSR markers [16,34,36-38]. However, the map was also saturated using newly developed SSR markers designed from the information of SSR sequences in the gene bank and by using other marker types such as SRAP, ISSR, EST-SSR and SCAR. These genetic maps will be a useful addition in understanding the Brassica napus genome and tagging the economically important genes in this important oil seed crop species.

\section{Plant Materials and DNA Extraction}

More than two hundred DH lines were developed by microspore

*Corresponding author: Muhammad Tahir Department of Plant Science, University of Manitoba, 222 Agriculture Building,66 Dafoe Road, Winnipeg Manitoba, Canada, Tel: 204 474-6076, Fax: 204 474-7528; E-mail: tahir@cc.umanitoba.ca

Received December 01, 2011; Accepted December 26, 2011; Published January 04, 2012

Citation: Geng J, Javed N, McVetty PBE, Li G, Tahir M (2012) An Integrated Genetic Map for Brassica napus Derived from Double Haploid and Recombinant Inbred Populations. Hereditary Genetics 1:103. doi:10.4172/2161-1041.1000103

Copyright: $\odot 2012$ Geng J, et al. This is an open-access article distributed under the terms of the Creative Commons Attribution License, which permits unrestricted use, distribution, and reproduction in any medium, provided the original author and source are credited. 
culture from an $\mathrm{F}_{1}$ hybrid generated from a cross between two Canadian canola (B. napus) cultivars called Polo and Topas. Randomly selected 190 fertile DH lines and two parents were used for the construction of a genetic map. One hundred and thirty-six F5 RI lines were developed through continuous self-pollination from the same cross between Polo and Topas. Randomly selected 94 RI lines and two parents were used for the construction of a second genetic map. Considering the differences in parental cultivars, populations were found to be segregating for various agronomic traits such as plant height, yield and oil content. Approximately 0.7 gram of young leaves from each greenhouse grown $\mathrm{DH}$ and RI lines were collected for genomic DNA extraction using a modified CTAB method $[9,10]$.

\section{Molecular markers}

Simple Sequence Repeats (SSR): The sequences of 387 public SSR primer pairs were obtained from published papers [16,34,37-39]. In addition, 130 unpublished sequences for SSR primer pairs, named SR+ hereafter, were kindly provided by the Molecular Genetics Laboratory at the University of Manitoba. Morever, new SSRs were also developed in this study. For this purpose, an online SSR identification tool called SSRIT (http://www.gramene.org/db/markers/ssrtool) was applied to detect the di-, tri-, tetra- SSR sequences in 4563 Brassica genome survey sequences downloaded from the NCBI website. The SSR primers were then designed using a Primer3Plus (http://www.bioinformatics. $\mathrm{nl} /$ cgi-bin/primer3plus/primer3plus.cgi) program with options to generate primers with a length of $18-22 \mathrm{bp}, \mathrm{GC}$ content of $45-55 \%$, and the predicted PCR products ranging from 100 to $500 \mathrm{bp}$. The newly developed SSR markers were named RaAC (represents Brassica rapa AC library), OlBH (reprents Brassica oleracea BH library) and NaJB (reprents Brassica napus JBnB BAC). Using the same tool, EST-SSR primers were detected and designed from sequences of Brassica ESTs downloaded from NCBI. The sequence information of SSR and ESTSSR is available in supplementary materials.

Sequence-Related Amplified Polymorphism (SRAP): The protocols to develop SRAP markers and sequences for primer pairs have been described previously $[9,10]$. The primers were kindly provided by the Molecular Genetics Laboratory at the University of Manitoba.

Intersimple Sequence Repeats (ISSR): The ISSRs are semiarbitrary markers developed by Zietkiewicz et al. [11], and consist of PCR amplification of DNA sequences delimited by two inserted microsatellites. The PCR amplifications are performed with only one primer composed of SSR units, with or without an anchoring end. Seventy seven ISSR primers were obtained from Nanjing Sunshine Biotechnology Co., Ltd, China while 116 ISSR primers were designed in our lab (details in supplementary materials).

Sequence-Characterized Amplified Region (SCAR): The published sequences of full length $B$. napus genes which are involved in fatty acid biosynthesis were used to design gene specific primers using Primer3Plus (http://www.bioinformatics.nl/cgi-bin/primer3plus/ primer3plus.cgi). The remaining sixteen primer sequences have been reported previously [40].

\section{Genotyping for molecular markers}

General Protocol: The DNA from individual lines in each of the mapping populations described earlier was used as a template to amplify the regions corresponding to various types of primers as described above. In order to separate the PCR products on the
ABI 3100 Genetic analyzer, a common 19-base sequence of M13 (CACGACGTTGTAAAACGAC) was added before the 5' end of forward primers. Four-color fluorescent dyed M13 primers (FAM-blue, VIC-green, NET-yellow PET-red) were synthesized by ABI company (Foster City, California). The PCR reactions were performed in 384well plates where each well contained $10 \mu \mathrm{l}$ of mixture containing $20 \mathrm{ng}$ of template DNA, $0.05 \mathrm{mM}$ forward primer, $0.15 \mathrm{mM}$ reverse primer, $0.1 \mathrm{mM}$ labed-M13 primer, $0.15 \mathrm{mM}$ dNTPs, $2.0 \mathrm{mM} \mathrm{MgCl}, 1 \times \mathrm{PCR}$ buffer, and 0.5 Units of Taq DNA polymerase. The PCR cycling was programmed as i) $94^{\circ} \mathrm{C}$ for $5 \mathrm{~min}$; ii) 5 cycles of $94^{\circ} \mathrm{C}$ for $50 \mathrm{~s}, 56^{\circ} \mathrm{C}$ for $50 \mathrm{~s}, 72^{\circ} \mathrm{C}$ for $1 \mathrm{~min}$, with a $0.8^{\circ} \mathrm{C}$ decrease in annealing temperature at each cycle; iii) $30 \mathrm{cycles}$ of $94^{\circ} \mathrm{C}$ for $50 \mathrm{~s}, 51^{\circ} \mathrm{C}$ for $50 \mathrm{~s}, 72^{\circ} \mathrm{C}$ for $1 \mathrm{~min}$; iv) an elongation step of $7 \mathrm{~min}$ at $72^{\circ} \mathrm{C}$. Samples from four different color (FAM-blue, VIC-green, NET-yellow PET-red) labeled primers were pooled together after running PCR reactions and $2.5 \mu \mathrm{l}$ of the pooled samples was added to a $5.5 \mu \mathrm{l}$ mixture of formamide and 500 LIZ size standard (Applied Biosystems, Foster City, California) and denatured at $95^{\circ} \mathrm{C}$ for 5 minutes. The plates containing the samples were loaded into the auto sampler of the ABI 3100 Genetic analyzer equipped with $36 \mathrm{~cm}$ 16-channel arrays with a 40 min running time. The array profiles were analyzed with Genscan (ABI) software and specific polymorphic loci were scored with Genographer software (). The ABI files were converted to gel-like images and scoring of polymorphic loci by the software was confirmed visually. Each of the polymorphic loci was scored as a dominant marker (only one band was scored for codominant bands based on the maternal parent). The final molecular markers were scored as a primer name followed by the size of the amplified DNA fragment or visual band on the gel image. For example, OlBH001-234 represents the primer OlBH001 which amplified a 234 base pair DNA fragment.

\section{Segregation analysis and map construction}

Markers that were reproducibly polymorphic between the parental lines were scored in the DH and RI populations. Goodness of fit to expected ratios (1:1) of segregation of the markers was estimated by chi-square test ( $\chi^{2}$ test) at significance $P<0.05$. Markers that deviated from the expected ratios were also incorporated into the linkage analysis. Linkage analysis and map construction were performed by using Joinmap 3.0 software [41], to assemble 19 linkage groups. The Kosambi map function [42], was used for converting recombination frequency into genetic distance with LOD values of 6 to 12 and a recombination rate of 0.4 . Linkage groups containing more than two common markers in each map were selected and integrated using the 'Combine the Groups for Map Integration' function.

\section{Results}

\section{Segregation analysis of molecular markers}

Segregation analysis of molecular markers showing detectable allelic variation or polymorphism between parents is an inevitable step in map construction. A total of 1013 primer pairs or primers, corresponding to various types (SSR, SRAP, ISSR and SCAR) of molecular markers were used in this study. Of these primers, 698 (68.9\%) amplified successfully, and were further used to screen polymorphism between parents. A total of 716 and 533 polymorphic bands were collected to construct genetic maps for the $\mathrm{DH}$ and RI populations, respectively. A test of goodness of fit to $1: 1$ ( $\chi^{2}$ test) revealed that $362(50.6 \%)$ and 174 (32.6\%) molecular markers (distorted markers) violated the expected Mendelian segregation ratio of $1: 1$ for the DH and RI populations, 
N01

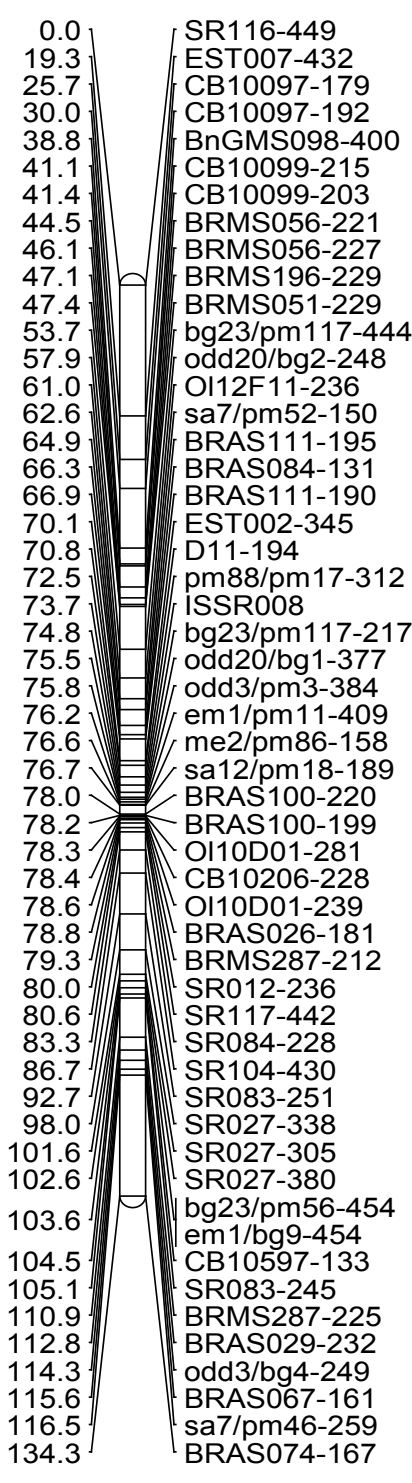

N02

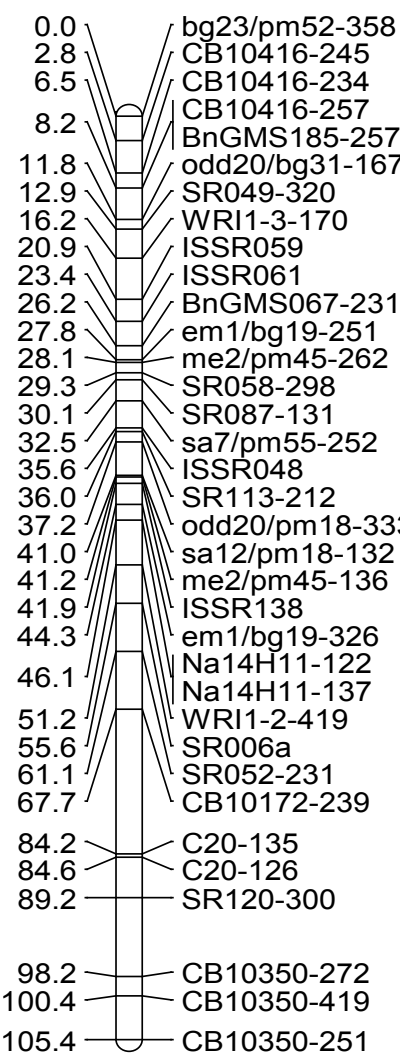

\section{N06}

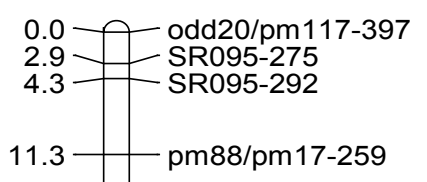

20.

\section{5}

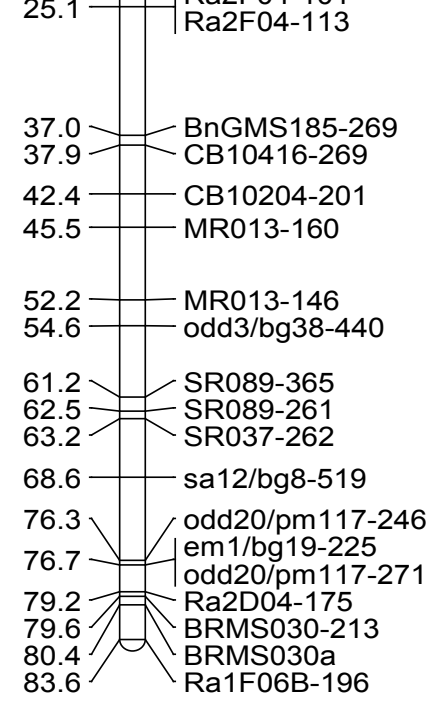

\section{N03}

$0.0 \quad$ BRMSO08-131 $\left.\begin{array}{r}5.0 \\ 16.3\end{array}\right] \quad$ Na10G10-182

$22.0]$.

Sa12/bg38-422

BRMSO71-

BRMS303-223

BRMS303-217

D03-203

D03-217

BnGMS079-353

BnGMS079-333

sa12/bg18-224

D21-420

64.1 1 年

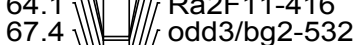

69.6 ||$=\mid$ sa12/pm7-262

73.7 ISSR055

82.7 $=$ Na10D03-164

87.1 $=$ CB10132-277

93.8 BRMS027-198

96.9 BRAS065-217

$101.0 \sqrt[B]{\text { BnGMS185-163 }}$

CB10403-162

$102.6 \_$CB10403-172

$109.0-\quad$ SR078-204

125.2 / BRMS043-240

$127.7 \checkmark$ BRMS042-152

127.9 BRMS042-124

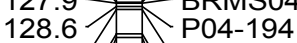

129.2 $=181$

134.1

134.8 odd3/bg1-129

140.2 odd $20 / \mathrm{bg} 2-262$

141.4 $=\left[\begin{array}{l}140.2 \\ \text { odd2/.pm } 18-388\end{array}\right.$

$\left.\begin{array}{l}141.4 \\ 141.6\end{array}\right]\left[\begin{array}{l}\text { sa12/pm18-388 } \\ \text { pm88/pm46-388 }\end{array}\right.$

$\left.\begin{array}{l}141.6 \\ 143.6\end{array}\right]\left[\begin{array}{l}p m 88 / p m 46-388 \\ m e 2 / p m 86-467\end{array}\right.$

149.1 - em1/pm4-238

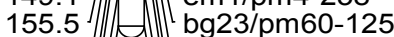

157.1

162.2 ||| ||

165.8 || $\mid$ BRMS048

176.1

182.9 ISSR185

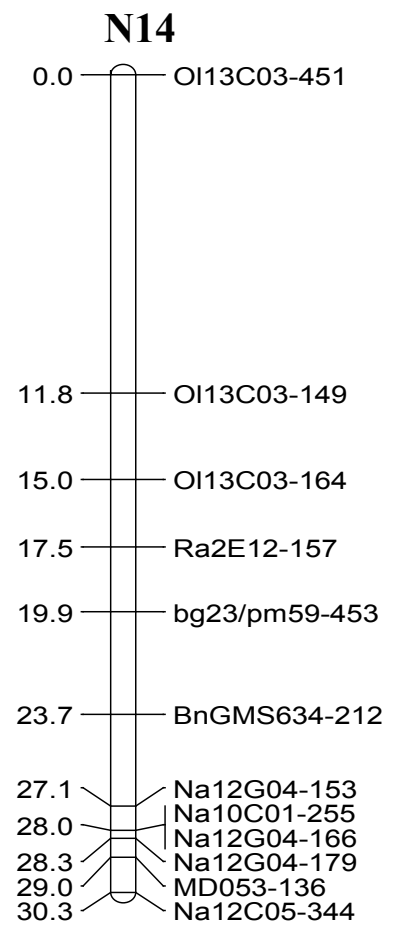

N04

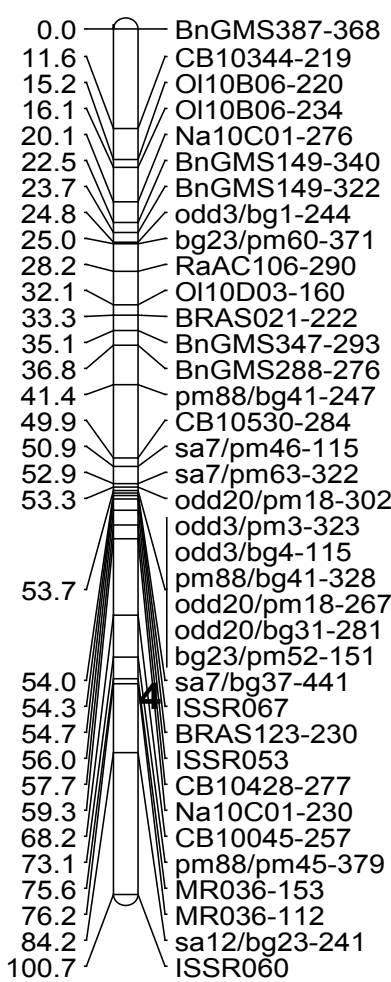

N05

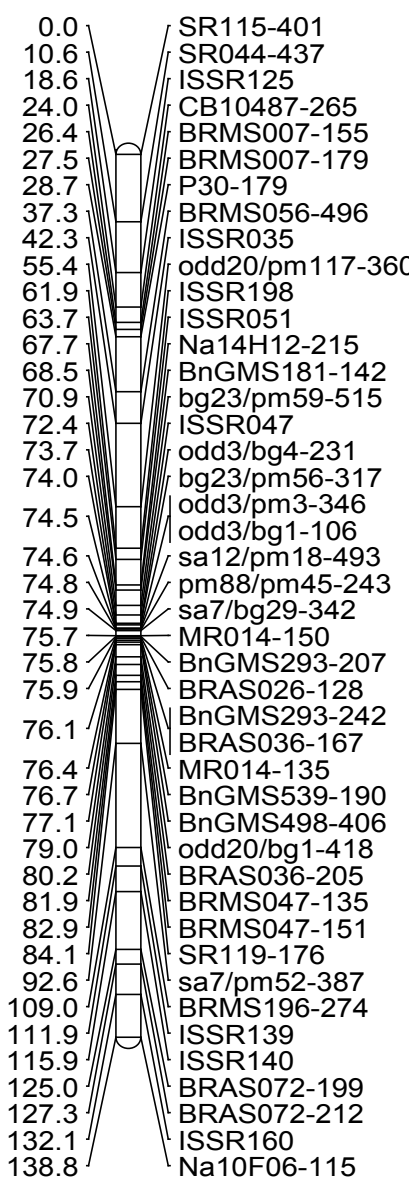

to be continued 


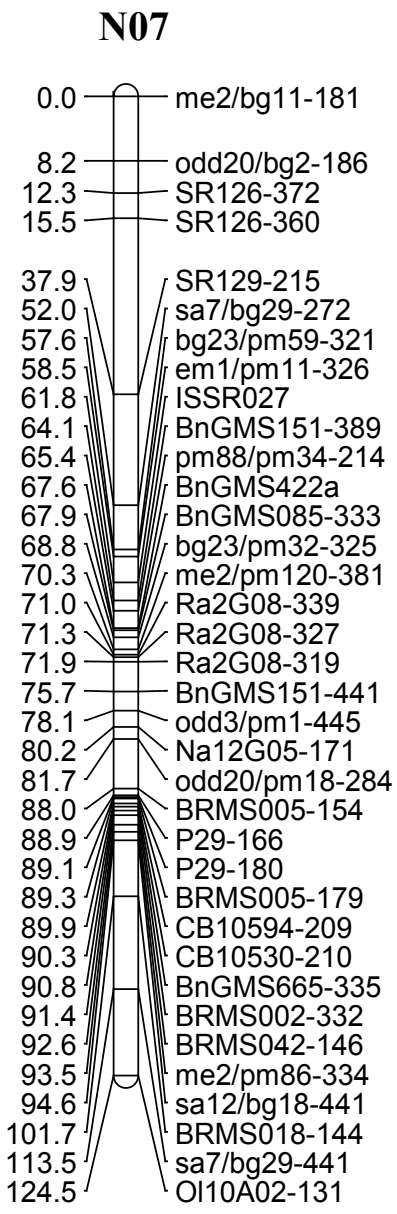

N11

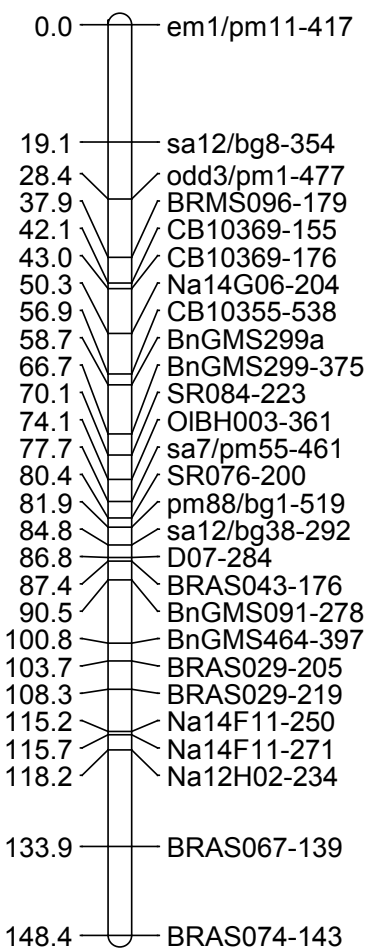

N08

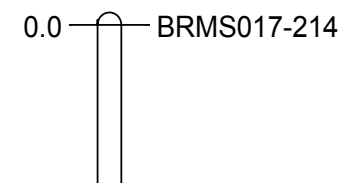

29.5 - BRMS027-208

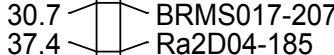

$39.5-$ Ra1F06B-206

Ni4A07-206

68.0 BRMS006-196

68.6

72.7

76.1 BRMS001-123

81.9

83.7 - SR055-446

84.2 $\sqrt{\text { CB10578-251 }}$

$84.8 \sqrt{\mathrm{P} 26-118}$

$85.5 \simeq$ em $1 / \mathrm{pm} 4-179$

90.3 $\quad$ pm88/pm17-472

90.9 位 ISSR127

92.0 / (

92.7 $-\mathrm{CB} 10364-248$

92.9 CB10364-224

94.4 BnGMS003-378

94.8 | SR092-131

95.3 sa7/pm63-297

95.7 - sa7/pm63-450

96.4 ( SR086-297

105.6

110.4 $=$ SR055-480

113.3 BnGMS177-354

126.2 SR050-291

135.9 SR065-285

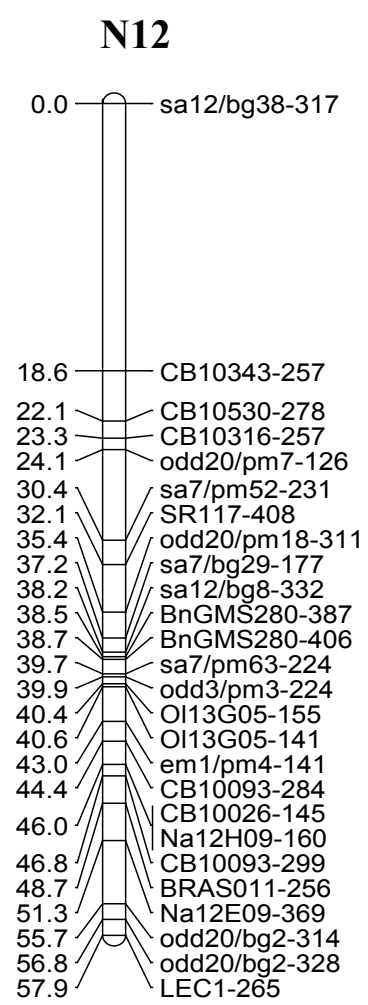

N09

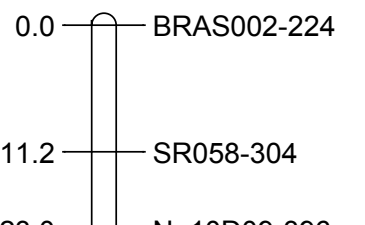

$\left.\begin{array}{l}23.0 \\ 23.9\end{array}\right) \quad / \quad \begin{aligned} & \mathrm{Na10D09}-396 \\ & \mathrm{Na10D09}-300\end{aligned}$

26.2 $=$ em1/bg19-299

26.7 em $1 / \mathrm{bg} 19-265$

30.6 SR058-310

32.3 BnGMS313-169

32.5 CB10347-190

33.1 C CB10347-183

33.9 sa7/pm55-145

$38.2 \Rightarrow$ ISSR155

40.0 - em $1 / \mathrm{bg} 9-254$

40.7 $\quad$ odd3/pm1-335

43.7 odd20/pm7-111

47.8 em $1 / \mathrm{pm} 4-164$

49.2 bg23/pm60-164

62.5 1 odd20/pm18-236

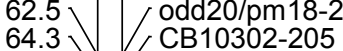

64.9 CB10302-196

$681>$ bg23/pm52-202

68.6 × $\ U S 3 R S S-212$

71.9 $\quad \mid \begin{aligned} & \text { em } 1 / \mathrm{bg} 19-493 \\ & \text { bg } 23 / \mathrm{pm} 117-483\end{aligned}$

74.2 SR029-243

74.7 SR052-247

$76.1 \quad$ SR028-308

$83.7 \_$SR091-266

\section{N10}

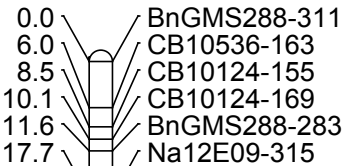

17.7 $=$ Na12E09-315

19.6 sa12/pm7-345

1.1 MR156-195

22.1 Ra2E07-163

22.5 odd3/bg1-256

22.8 odd3/bg38-305

23.1 $m$ me2/pm79-481

24.4 Na12C05-351

25.2 $=\mid$ MR156-209

26.6 - BnGMS625-360

28.3 BRMS046-96

32.9 EST001-435

39.1 em1/pm4-157

39.5 - bg23/pm60-157

42.9 - ISSR031

49.2 bg23/pm52-216

51.1

56.8 OIAJ120-360

60.9 - sa7/bg37-430

65.9 em $1 / \mathrm{bg} 9-406$

69.1 $\quad$ EST008-190

72.9 CB10079-160

77.7 Ol10B03-100

81.1 $/$ odd20/bg31-483

83.7 \CB10079-172

90.9 $1 /$ em $1 /$ pm11-250

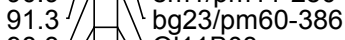

98.3 Ol11B03a

FAE02-211

$116.7-$ BRAS069-204

\section{N15}

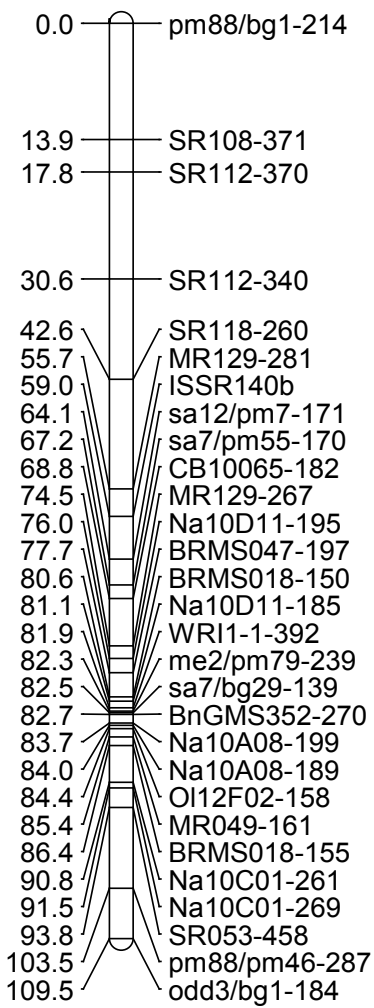

N16

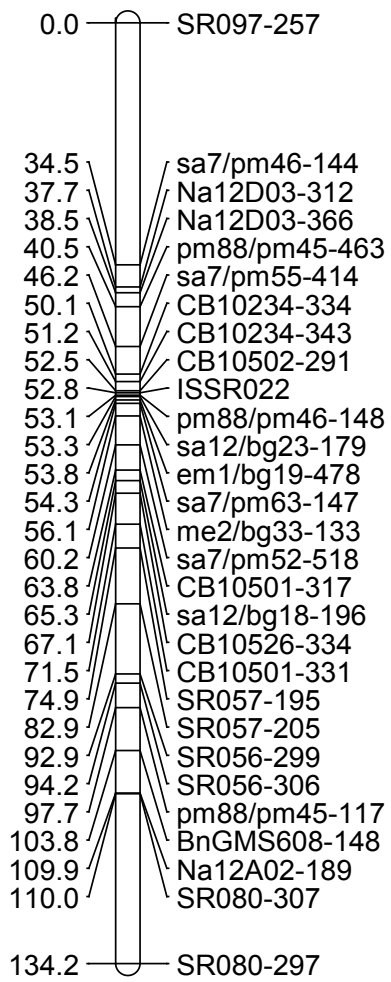


Citation: Geng J, Javed N, McVetty PBE, Li G, Tahir M (2011) An Integrated Genetic Map for Brassica napus Derived from Double Haploid and Recombinant Inbred Populations. Hereditary Genetics 1:103. doi:10.4172/2161-1041.1000103

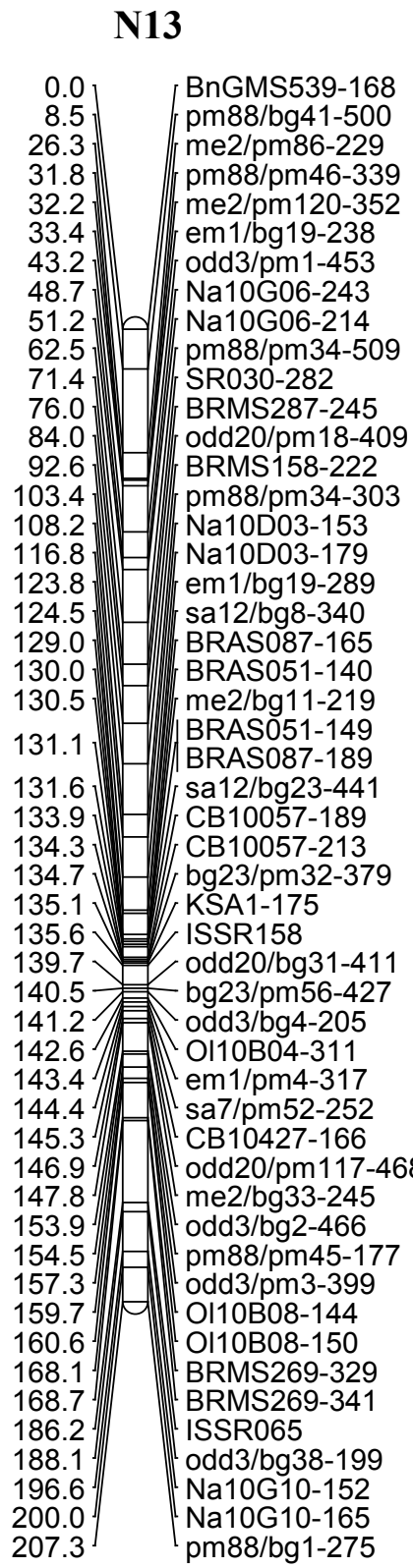

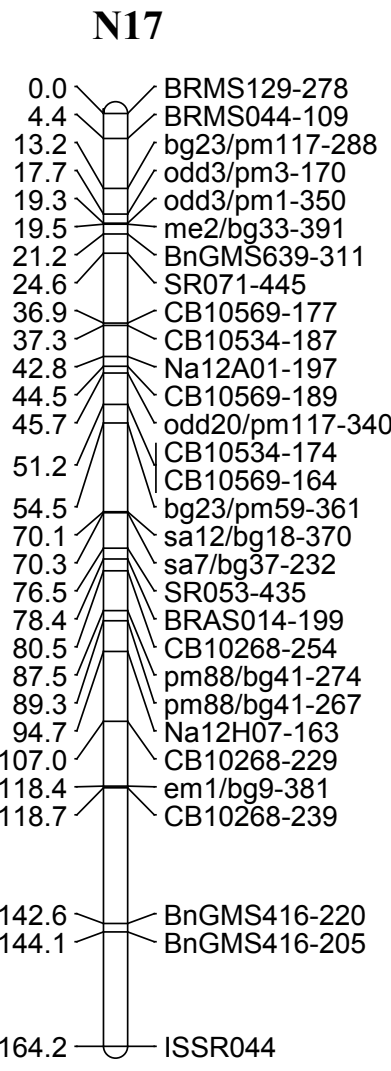

N18

N19

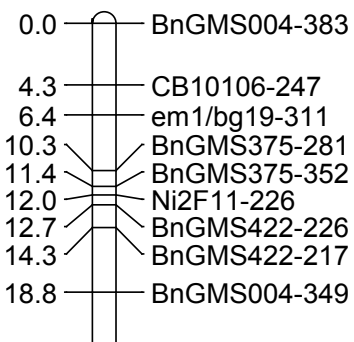

$23.6-\quad-e m 1 / b g 1-409$

|

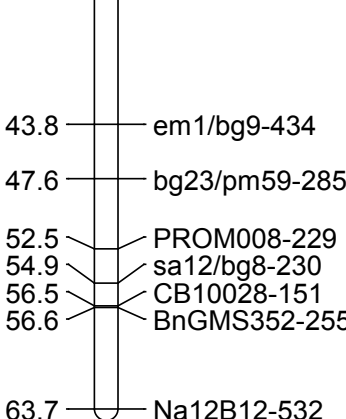

\section{$0.0-$ CB10344-202 \\ $2.7-$ SR043-342 \\ $7.4-$ CB10350-220 \\ $15.0-$ BnFUS3\#2-1-91}

$20.4-$ CB10355-218

26.2 BnGMS213-126

28.8 Na12D04-300

$30.6-\operatorname{RaAC} 101-171$

$34.4-\mathrm{Na12D04-296}$

41.4 Na12E03-286

41.7 SR090-182

42.6 1 BnFUS3\#2-5-329

47.1 WRI1-4-265

$50.3>$ CB10524-235

51.5 BnLEC2-3-265

55.3 CB10471-186

56.2 LEC1-334

58.9 Ra2F11-246

62.8 1 CB10597-117

65.1 BnLEC2-1-301

72.5 CB10524-224

78.3 WRI1-5-158

80.9 LEC1-325

86.8 Na12G05-118

90.6 BRMS054-148

99.3 - CB10415-196

108.7 NaFM100-157

109.6 CB10027-238

$122.1-$ em $1 / \mathrm{bg} 19-438$

Figure 1: A DH-based genetic map for Brassica napus.

respectively (details in supplementary materials). Among these 362 distorted markers in the $\mathrm{DH}$ population, 175 (48.3\%) markers biased to the maternal parent Polo, and 187 (51.7\%) markers showed bias to the paternal parent Topas, however, the second $\chi^{2}$ test on distorted markers resulted in a perfect fit to a Mendelian segregation ratio of 1:1, which indicates that the $\mathrm{DH}$ population was a nomal population. Whereas, among the 174 distorted markers of the RI population, 113 (64.9\%) markers biased to the maternal parent Polo, and 71 (35.1\%) markers biased to the paternal parent Topas, the result of second $\chi^{2}$ test showed a significant violation to Mendelian segregation ratio of 1:1.

\section{Construction of individual genetic maps}

The data collected from the genotyping for all the polymorphic molecular markers were used for linkage analysis and map construction.
Out of 716 molecular markers in the DH population, a total of 620 (87.0\%) molecular markers, including 383 SSR, 191 SRAP, 29 ISSR and 17 SCAR markers were assigned onto 19 linkage groups (LGs). Using common SSR markers from existing Brassica reference linkage maps $(16,34)$ the LGs were anchored to chromosomes named N01 to N19 (Figure 1). This map covered $2244.1 \mathrm{cM}$ with an average marker spacing of $3.6 \mathrm{cM}$. The length of each LG ranged from $30.3 \mathrm{cM}$ to $207.3 \mathrm{cM}$ for $\mathrm{N} 14$ and N13, respectively, the average marker spacing ranged from 2.2 to $5.5 \mathrm{cM}$ for N12 and N11, respectively. The overall number of markers on each chromosome ranged from 12 to 53 for N14 and N01, respectively. Individually, SSR markers ranged from 11 to 38 , SRAP markers ranged from 1 to 26,29 ISSR markers were mapped onto 13 chromosomes, ranged from 1 to 8 and 17 SCAR markers were mapped onto 8 chromosomes, ranged from 1 to 8 (Table 1). 
Citation: Geng J, Javed N, McVetty PBE, Li G, Tahir M (2011) An Integrated Genetic Map for Brassica napus Derived from Double Haploid and Recombinant Inbred Populations. Hereditary Genetics 1:103. doi:10.4172/2161-1041.1000103

Page 6 of 16

\begin{tabular}{|c|c|c|c|c|c|c|c|}
\hline LGs & Total Markers & SSR & ISSR & SRAP & SCAR & Length (cM) & Marker density (cM/marker) \\
\hline N01 & 53 & 38 & 1 & 14 & - & 134.3 & 2.5 \\
\hline N02 & 35 & 20 & 4 & 9 & 2 & 105.4 & 3.0 \\
\hline N03 & 46 & 31 & 3 & 12 & - & 182.9 & 4.0 \\
\hline N04 & 37 & 19 & 3 & 15 & - & 100.7 & 2.7 \\
\hline N05 & 44 & 25 & 8 & 11 & - & 138.8 & 3.2 \\
\hline N06 & 24 & 16 & - & 8 & - & 83.6 & 3.5 \\
\hline N07 & 36 & 22 & 1 & 13 & - & 124.5 & 3.5 \\
\hline N08 & 31 & 24 & 2 & 5 & - & 135.9 & 4.4 \\
\hline N09 & 29 & 14 & 1 & 12 & 2 & 83.7 & 2.9 \\
\hline N10 & 35 & 20 & 1 & 13 & 1 & 116.7 & 3.3 \\
\hline N11 & 27 & 21 & - & 6 & - & 148.4 & 5.5 \\
\hline N12 & 26 & 14 & - & 11 & 1 & 57.9 & 2.2 \\
\hline N13 & 51 & 22 & 2 & 26 & 1 & 207.3 & 4.1 \\
\hline N14 & 12 & 11 & - & 1 & - & 30.3 & 2.5 \\
\hline N15 & 29 & 20 & 1 & 7 & 1 & 109.5 & 3.8 \\
\hline N16 & 29 & 17 & 1 & 11 & - & 134.2 & 4.6 \\
\hline N17 & 30 & 18 & 1 & 11 & - & 164.2 & 5.5 \\
\hline N18 & 17 & 11 & - & 5 & 1 & 63.7 & 3.7 \\
\hline N19 & 29 & 20 & - & 1 & 8 & 122.1 & 4.2 \\
\hline Total & 620 & 383 & 29 & 191 & 17 & 2244.1 & 3.7 \\
\hline Average & 32.6 & 20.2 & 2.2 & 10.1 & 0.9 & 118.1 & 3.7 \\
\hline
\end{tabular}

Table 1: Main characteristics of DH-based genetic map for Brassica napus.

\begin{tabular}{|c|c|c|c|c|c|c|}
\hline LGs & Total markers & SSR & ISSR & SCAR & Length (cM) & Marker density (cM/marker) \\
\hline N01 & 43 & 43 & - & - & 115.4 & 2.7 \\
\hline N02 & 9 & 9 & - & - & 71.3 & 7.9 \\
\hline N03 & 19 & 17 & 2 & - & 103.1 & 5.4 \\
\hline N04 & 36 & 30 & 6 & - & 103.8 & 2.9 \\
\hline N05 & 23 & 17 & 6 & - & 132.0 & 5.7 \\
\hline N06 & 13 & 12 & 1 & - & 47.0 & 3.6 \\
\hline N07 & 20 & 19 & 1 & - & 95.6 & 4.8 \\
\hline N08 & 11 & 11 & - & - & 43.4 & 3.9 \\
\hline N09 & 17 & 13 & 1 & 3 & 95.1 & 5.6 \\
\hline N10 & 10 & 10 & - & - & 52.8 & 5.3 \\
\hline N11 & 21 & 19 & 2 & - & 108.3 & 5.2 \\
\hline $\mathrm{N} 12$ & 19 & 15 & 2 & 2 & 112.9 & 5.9 \\
\hline N13 & 25 & 22 & 2 & 1 & 107.8 & 4.3 \\
\hline N14 & 15 & 14 & 1 & - & 97.1 & 6.5 \\
\hline N15 & 17 & 16 & 1 & - & 53.2 & 3.1 \\
\hline N16 & 18 & 16 & 2 & - & 54.2 & 3.0 \\
\hline N17 & 12 & 12 & - & - & 112.6 & 9.4 \\
\hline N18 & 13 & 13 & - & - & 82.0 & 6.3 \\
\hline N19 & 8 & 8 & - & - & 62.6 & 7.8 \\
\hline Total & 349 & 316 & 27 & 6 & 1650.2 & 4.7 \\
\hline Average & 18.4 & 16.6 & 1.4 & 0.3 & 86.9 & 4.7 \\
\hline
\end{tabular}

Table 2: Main characteristics of RI-based genetic map for Brassica napus.

For the RI population, a total of 349 (65.5\%) molecular markers, including 316 SSR, 27 ISSR and 6 SCAR markers were assembled onto 19 LGs, and similar public SSR markers as for the DH population was used to anchor LGs to chromosomes (Figure 2). The average distance between markers and the total genome coverage were $4.7 \mathrm{cM}$ and $1649.1 \mathrm{cM}$, respectively. The length of each LG ranged from $43.4 \mathrm{cM}$ to $131.9 \mathrm{cM}$ for N08 and N05, respectively, the average marker spacing ranged from 2.7 to $9.4 \mathrm{cM}$ for N01 and N17, respectively. The total number of markers on each chromosome ranged from 8 to 43 for N19 and N01, repectively. Individually, SSR markers ranged from 8 to 43, ISSR markers were mapped onto 12 chromosomes, ranged from 1 to 6 and SCAR markers were mapped onto N09, N11 and N12 with numbers of 3, 2 and 1 , respectively (Table 2 ).

\section{Construction of an integrated genetic map}

Allele data sets related to the same LGs with at least two loci in common were integrated into one data set by applying Joinmap software. Common markers, ranging from 2 to 23 with an average of 9.6 were detected in the same linkage groups of the DH and RI populations, which allowed the construction of an integrated genetic map. A total number of 796 markers, including 539 SSR, 193 SRAP, 45 ISSR and 19 SCAR markers were combined into an integrated genetic map. This integrated genetic map comprised of 19 LGs and covered $2464.9 \mathrm{cM}$ with a marker density of $3.1 \mathrm{cM}$ per marker (Figure 3 ). The length of each LG ranged from $83.7 \mathrm{cM}$ to $209.4 \mathrm{cM}$ for N14 and N13, respectively. The number of markers on each LG ranged from 18 to 73 


\section{N01}

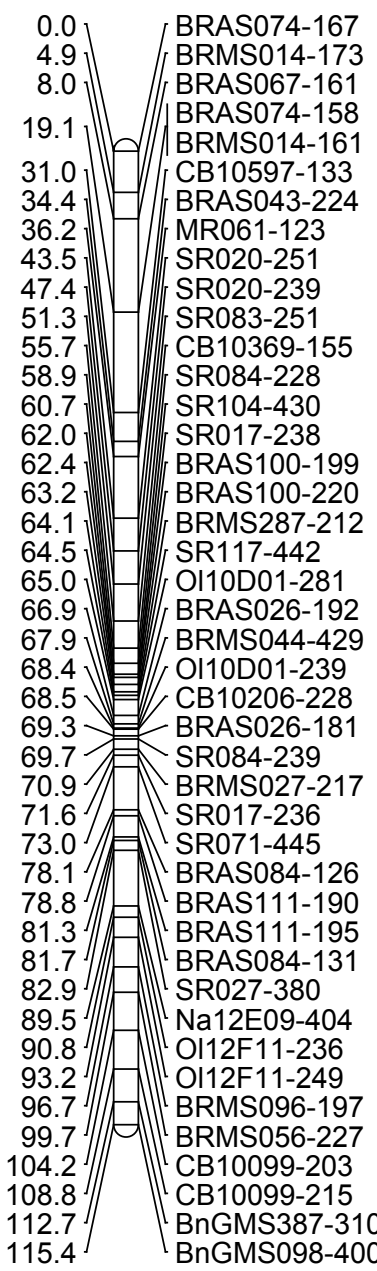

N08

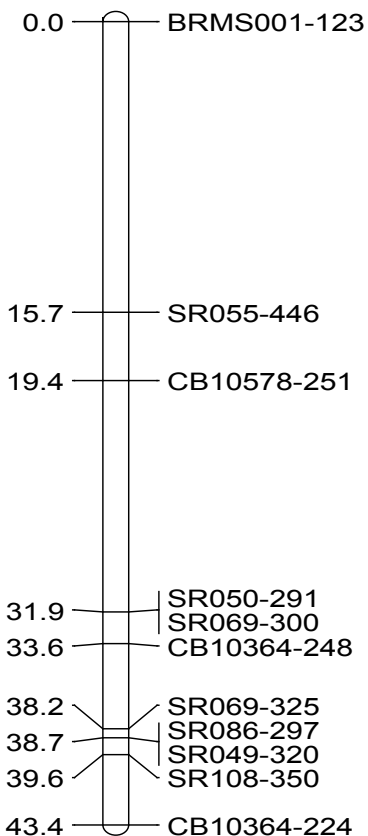

N02

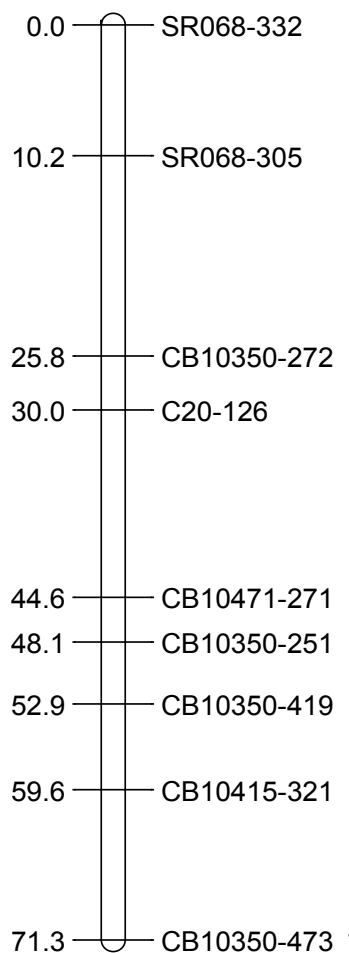

97.2

N03

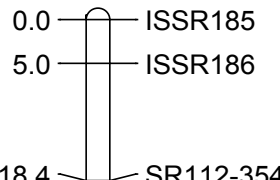

21.972510 23.17 BRAS050-159

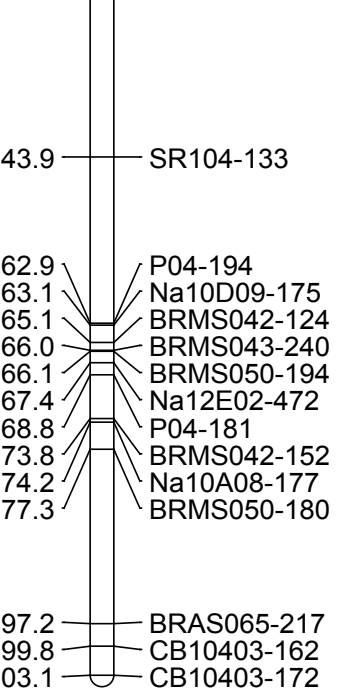

N05

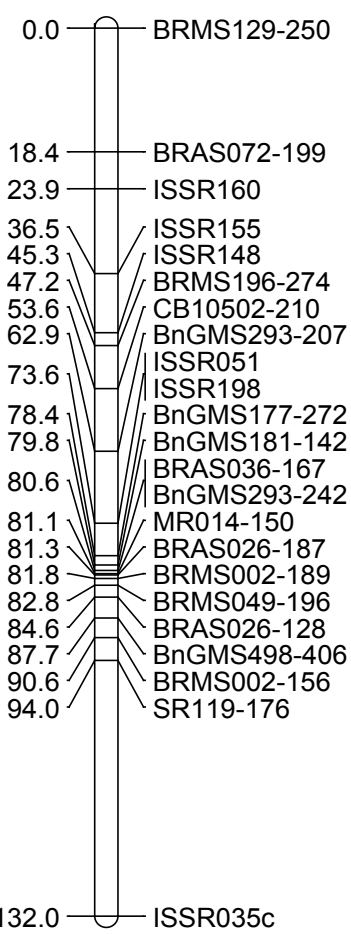

N06

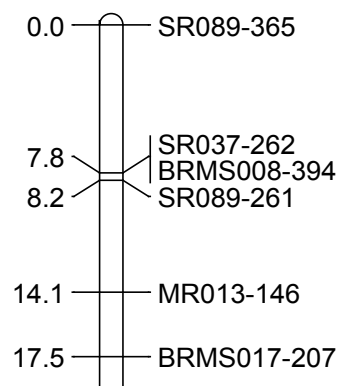

\section{N04}

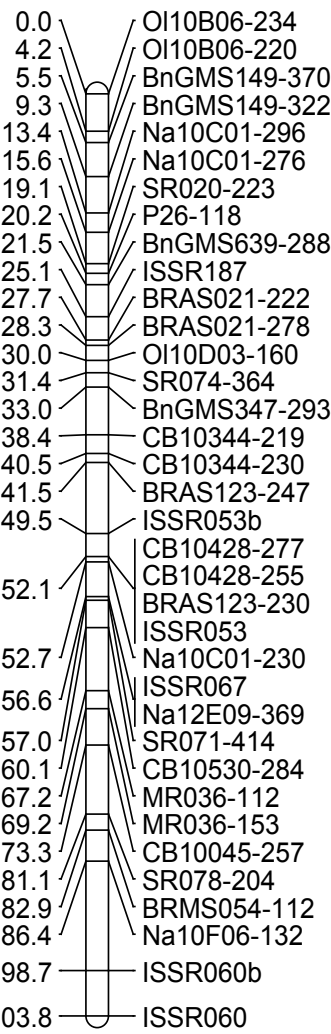

N07

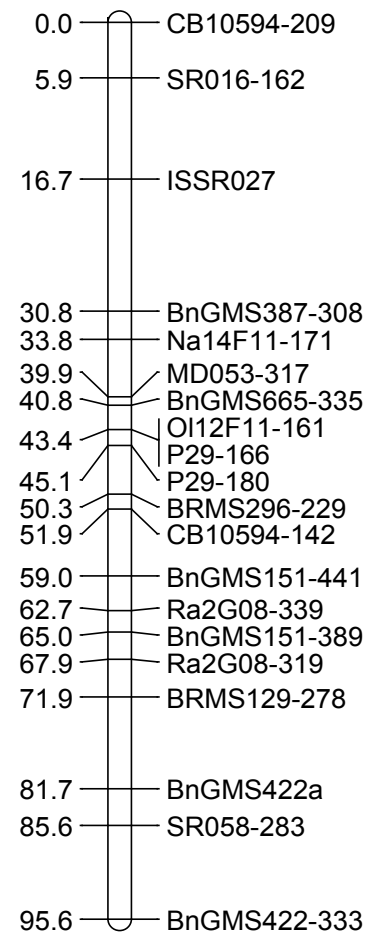


N09

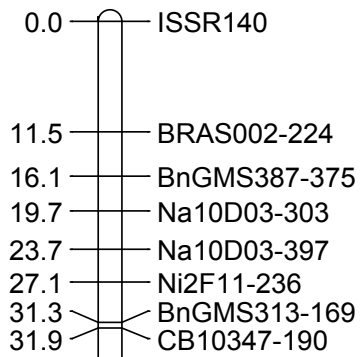

$31.9>$ CB10347-190

$37.0-12 \mathrm{Ni} F 11-226$

45.6 1 MCAT-214

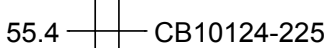

64.4 1 FUS3RSS-212

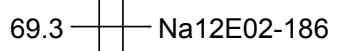

79.8 - SR091-266

$83.8-\quad$ SR029-243

$86.2-$ SR028-308

$95.1-$ LEC2-w-127

N13

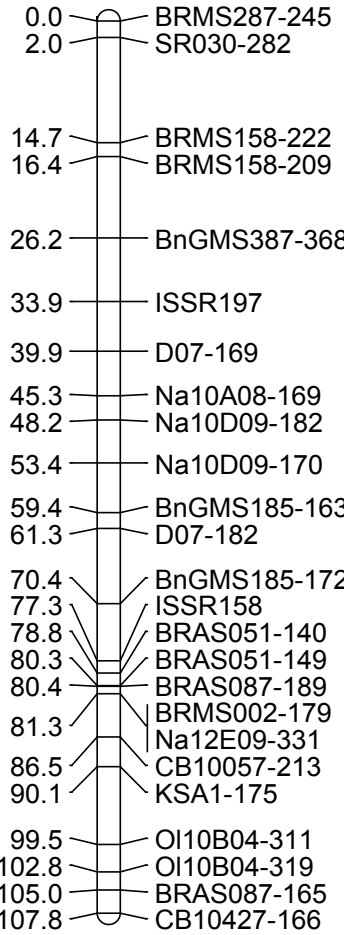

N10

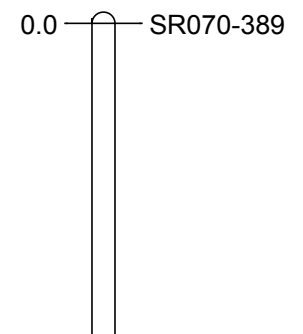

$19.8-$ BnGMS625-360

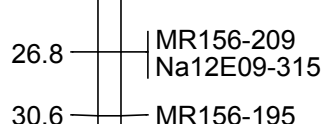

$30.6-\quad$ MR156-195

$32.1-\quad$ Na12C05-351

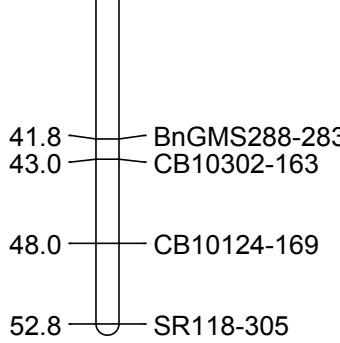

$52.8-$ SR118-305

N14

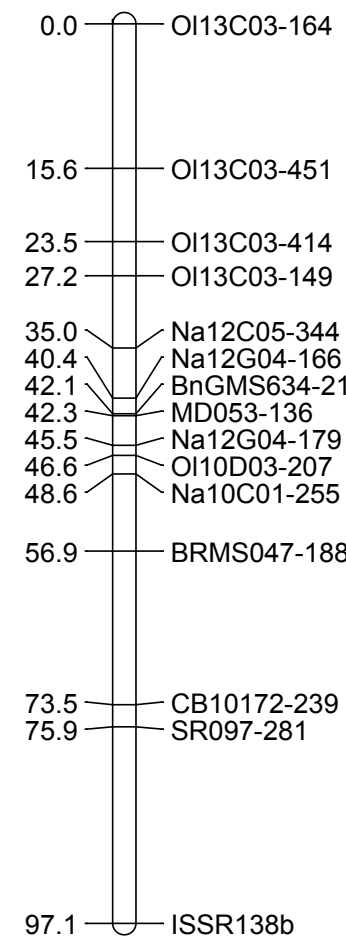

N11

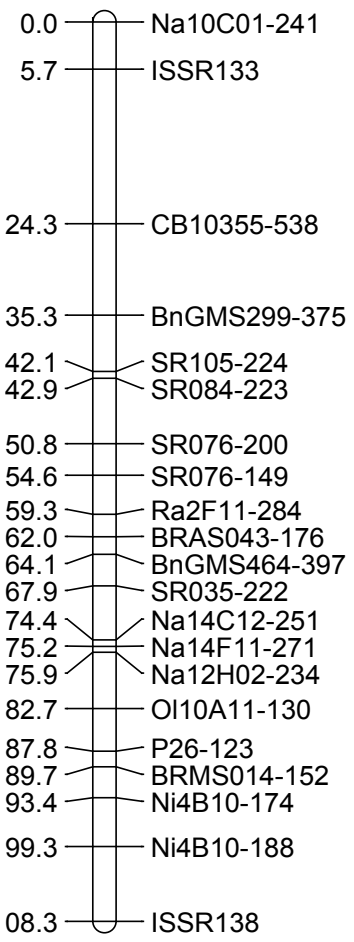

N12

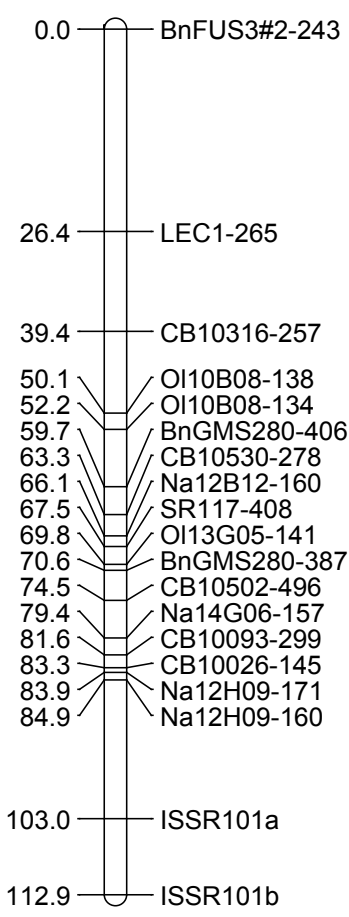

N16

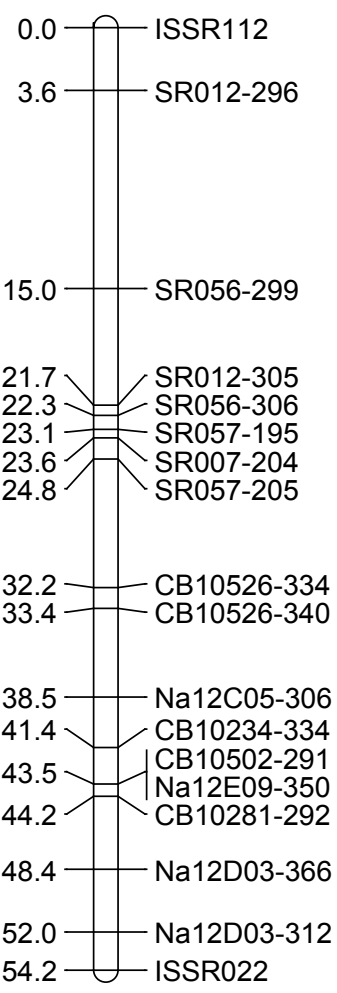




\section{N17}

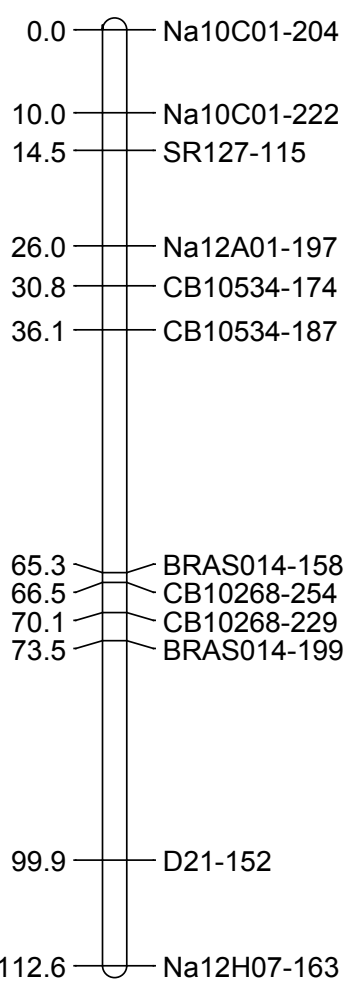

N18

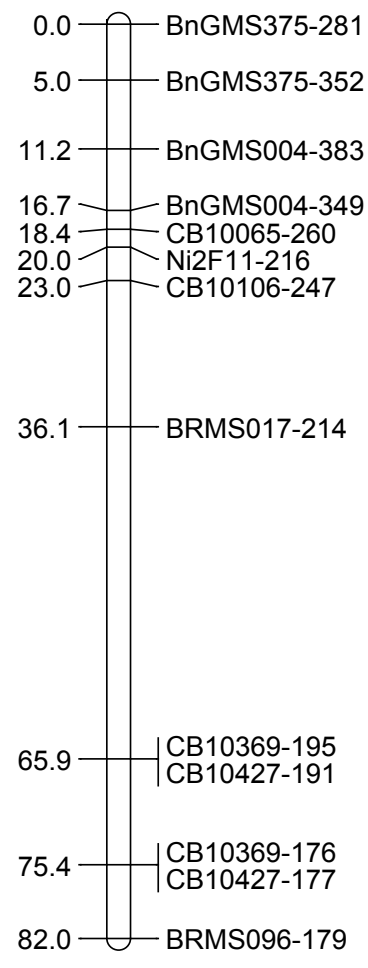

N19

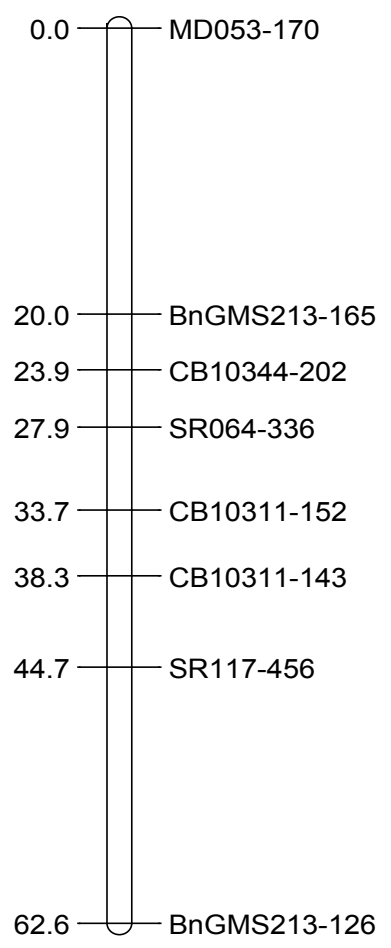

Figure 2: A RI-based genetic map for Brassica napus.

\begin{tabular}{|c|c|c|c|c|c|c|c|c|}
\hline LGs & Total markers & $\begin{array}{l}\text { Common } \\
\text { markers* }\end{array}$ & SSR & ISSR & SRAP & SCAR & Length (cM) & Marker density (cM/marker) \\
\hline N01 & 73 & 23 & 58 & 1 & 14 & - & 137.0 & 1.9 \\
\hline N02 & 40 & 4 & 25 & 4 & 9 & 2 & 129.1 & 3.2 \\
\hline N03 & 54 & 11 & 39 & 3 & 12 & - & 184.7 & 3.4 \\
\hline N04 & 55 & 18 & 34 & 6 & 15 & - & 104.1 & 1.9 \\
\hline N05 & 54 & 13 & 32 & 11 & 11 & - & 153.9 & 2.9 \\
\hline N06 & 32 & 5 & 23 & 1 & 8 & - & 84.0 & 2.6 \\
\hline N07 & 46 & 10 & 32 & 1 & 13 & - & 134.6 & 2.9 \\
\hline N08 & 45 & 7 & 36 & 2 & 7 & - & 130.6 & 2.9 \\
\hline N09 & 38 & 8 & 21 & 2 & 12 & 3 & 101.9 & 2.7 \\
\hline N10 & 38 & 7 & 23 & 1 & 13 & 1 & 117.4 & 3.1 \\
\hline N11 & 40 & 8 & 32 & 2 & 6 & - & 146.0 & 3.7 \\
\hline N12 & 35 & 10 & 20 & 2 & 11 & 2 & 94.0 & 2.7 \\
\hline N13 & 64 & 12 & 34 & 3 & 26 & 1 & 209.4 & 3.3 \\
\hline N14 & 18 & 9 & 16 & 1 & 1 & - & 83.7 & 4.7 \\
\hline N15 & 32 & 14 & 22 & 1 & 7 & 1 & 104.6 & 3.3 \\
\hline N16 & 37 & 10 & 24 & 2 & 11 & - & 108.9 & 2.9 \\
\hline N17 & 35 & 7 & 23 & 1 & 11 & - & 163.3 & 4.7 \\
\hline N18 & 25 & 5 & 19 & - & 5 & 1 & 121.9 & 4.9 \\
\hline N19 & 35 & 2 & 26 & - & 1 & 8 & 155.8 & 4.5 \\
\hline Total & 796 & 183 & 539 & 44 & 193 & 19 & 2464.9 & 3.1 \\
\hline Average & 41.9 & 18.1 & 54.1 & 4.6 & 19.3 & 4.1 & 129.7 & 3.1 \\
\hline
\end{tabular}

${ }^{*}$ Common markers represent the markers which are common to DH and RIL maps

Table 3: Main characteristics of integrated genetic map for Brassica napus. 


\section{N01}

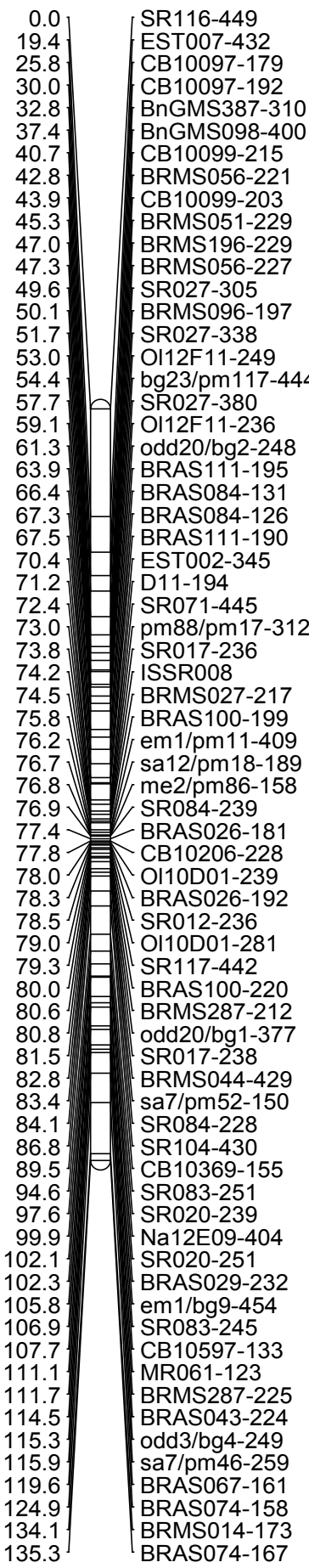

\section{N03}

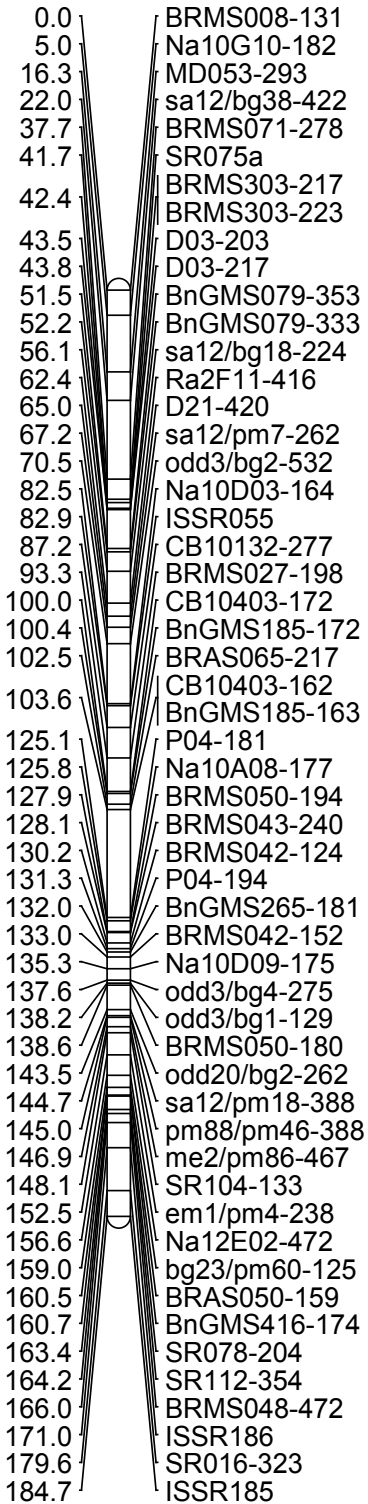

\section{N04}

\begin{tabular}{|c|c|}
\hline $\left.\begin{array}{c}0.0 \\
12.6 \\
14.3 \\
17.1 \\
19.7 \\
19.8 \\
22.1 \\
23.3 \\
24.4 \\
24.6 \\
24.8 \\
26.6 \\
27.9 \\
28.4 \\
29.2 \\
29.4 \\
32.3 \\
33.6 \\
35.5 \\
36.2 \\
37.0 \\
37.4 \\
41.7 \\
42.4 \\
44.8 \\
50.9 \\
52.0 \\
53.2 \\
53.9 \\
54.2 \\
54.7\end{array}\right)$ & 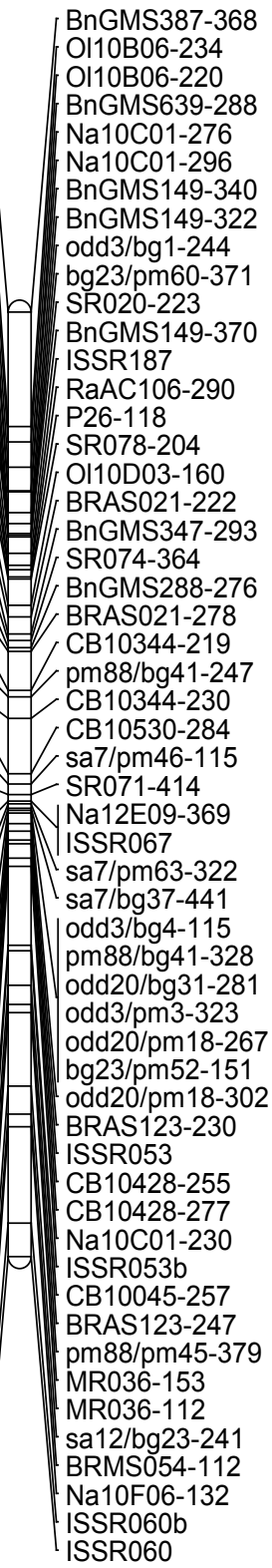 \\
\hline
\end{tabular}

N02

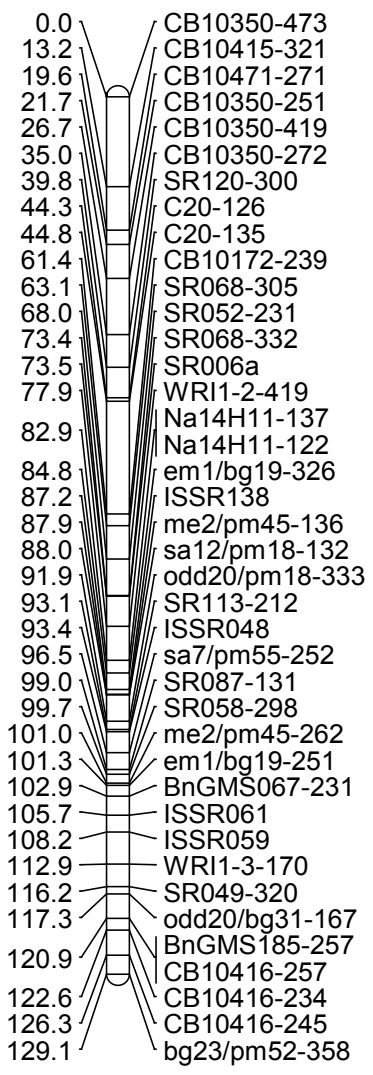

N06

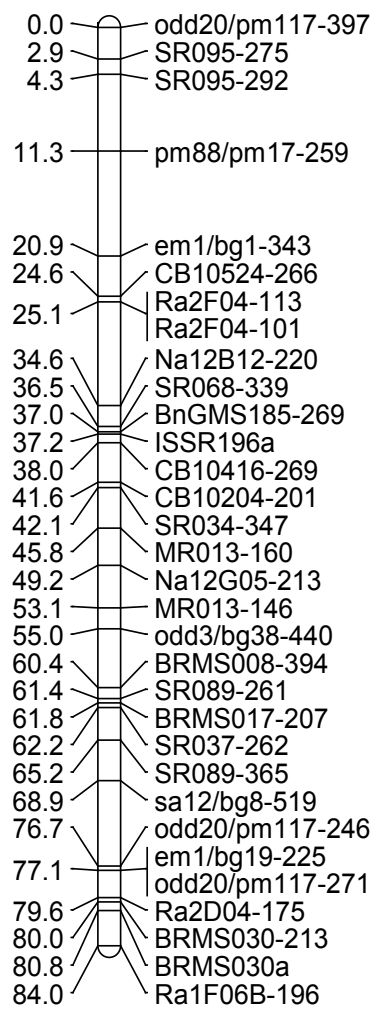


Citation: Geng J, Javed N, McVetty PBE, Li G, Tahir M (2011) An Integrated Genetic Map for Brassica napus Derived from Double Haploid and Recombinant Inbred Populations. Hereditary Genetics 1:103. doi:10.4172/2161-1041.1000103

Page 11 of 16

\section{N05}

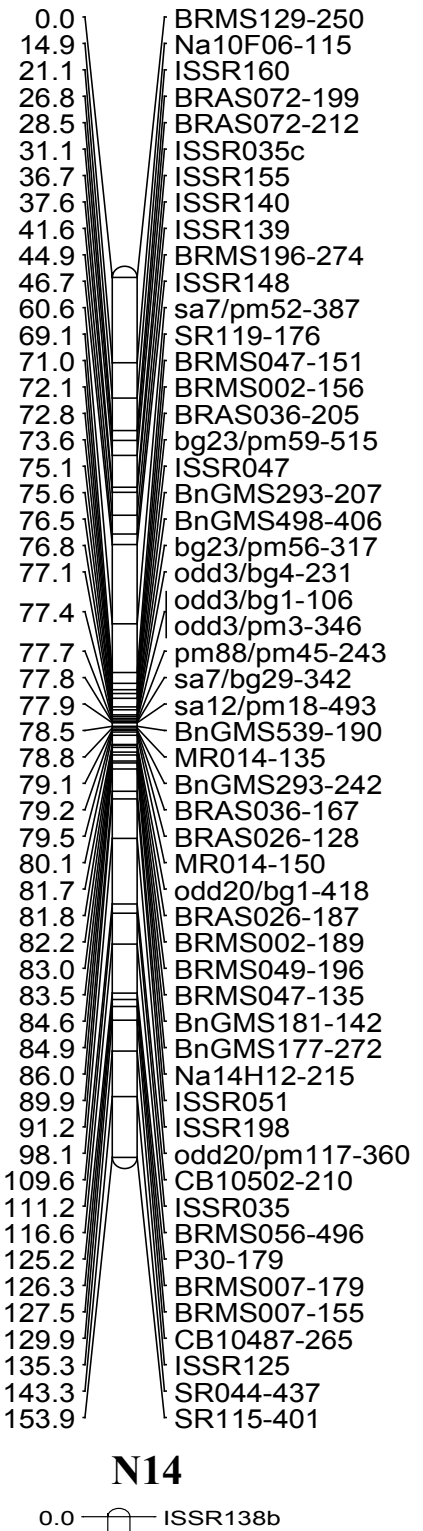

N07

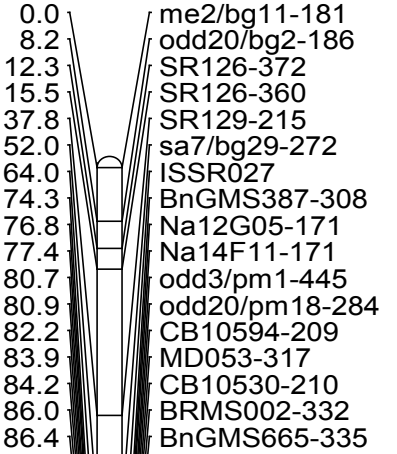

106.5

107.

109.0

112.5

113.5

114.5

116.7
117.6 117.6

126.5

134.2
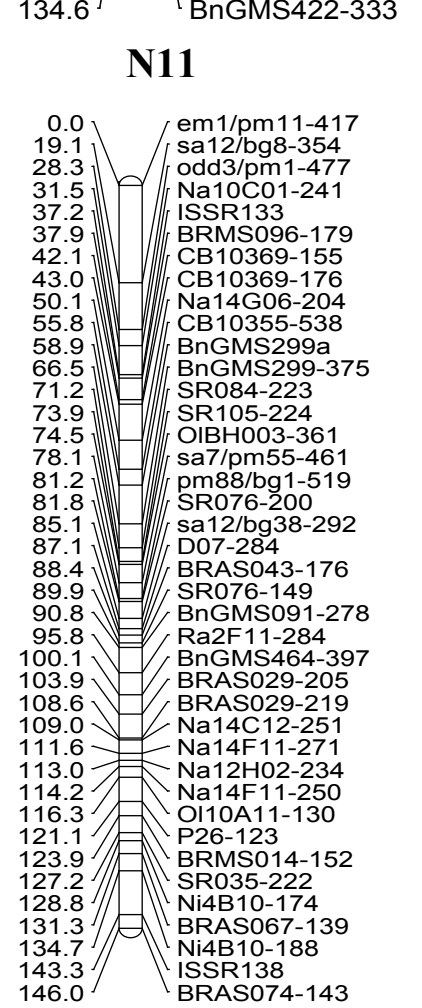

(1)

BnGMS422a

BnGMS151-441

sa7/bg29-441

BRMS129-278

bg23/pm59-321

Ol10A02-131

SR058-283

SR016-162

\section{N08}

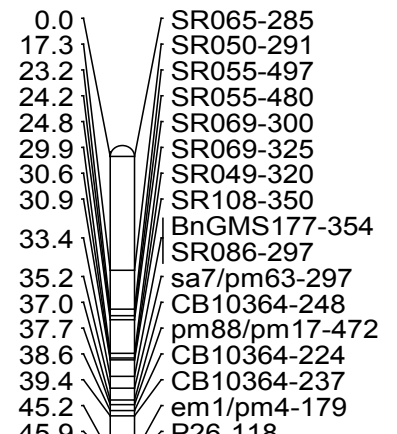

$46.6=$ CB10578-251

$47.7 \Rightarrow$ SR055-446

51.1 = sa7/pm46-246

$\left.\begin{array}{l}51.1 \\ 51.9\end{array}\right]$ ( sa7/pm63-450

52.3]

55.8 ] ISSR124

56.5

58.2

$\begin{aligned} & 58.2 \\ & 62.2\end{aligned} \mid$ SR062-260

62.7 $=$ IRMS006-196

87.9 $\Rightarrow$ Ni4A07-206

89.5 - em1/bg1-409

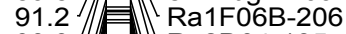

93.3 - Ra2D04-185

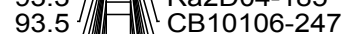

98.1 - BnGMS375-281

99.3 BnGMS375-352

99.8

100.0

100.4 BnGMS422-226

101.2 $\Rightarrow$ BRMS027-208

101.7 BnGMS422-217

105.5 BnGMS004-349

107.4

$\left.\begin{array}{l}112.7 \\ 130.6\end{array}\right] \begin{aligned} & \text { BnGMSO04-383 } \\ & \text { BRMS017-214 }\end{aligned}$

\section{N12}

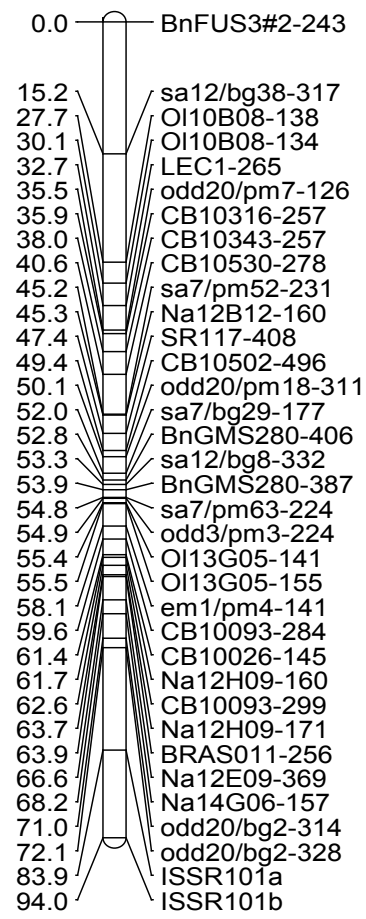

\section{N09}

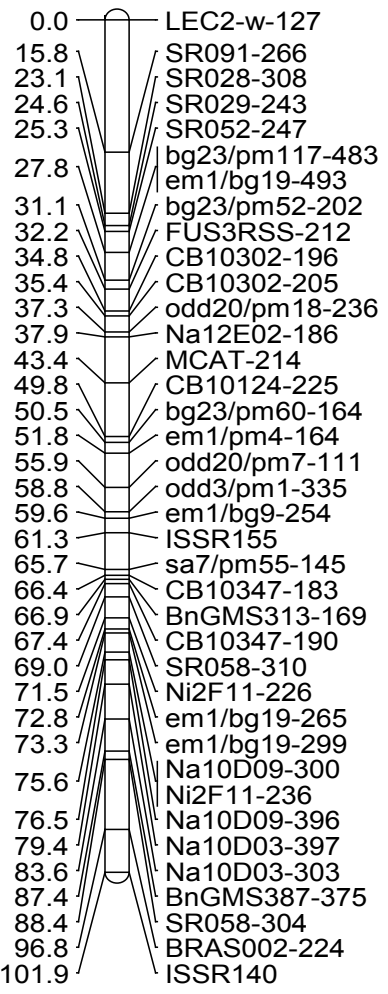

\section{N10}

\subsection{SR118-305}

0.4 BnGMS288-311

6.2 $\bigcirc$ CB10536-163

7.9 $\mathrm{CB} 10302-163$

$8.7-$ CB10124-155

9.4 CB10124-169

11.9 BnGMS288-283

15.8 EST001-435

19.6 Na12E09-315

21.0 a sa12/pm7-345

22.2 MR156-209

23.7 MR156-195

24.0 - $\begin{aligned} & \mathrm{R} \text { Ra2E07-163 } \\ & \text { odd } 3 / \mathrm{bg} 1-256\end{aligned}$

24.8 odd $3 / \mathrm{bg} 1-256$

25.1 me2/pm79-481

26.5 $\quad$ Na12C05-351

28.9

29.9 BRMS046-96

39.9 -1 em 1/pm4-157

43.7 - ISSR031

49.9 bg23/pm52-216

51.8 sa7/bg37-293

51.8 sa7/bg37-293

57.6 \# OIAJ120-360

61.6 Na7/bg37-430

66.6 em $1 /$ bg $9-406$

69.7 $=$ EST008-190

73.6 CB10079-160

78.4 OІ10B03-100

81.7 - odd20/bg31-483

84.3

91.6 em1/pm11-250

92.0 $\quad$ bg23/pm60-386

98.9 $/$ Ol11B03a

104.6
117.4 FAE02-211 


\section{N13}

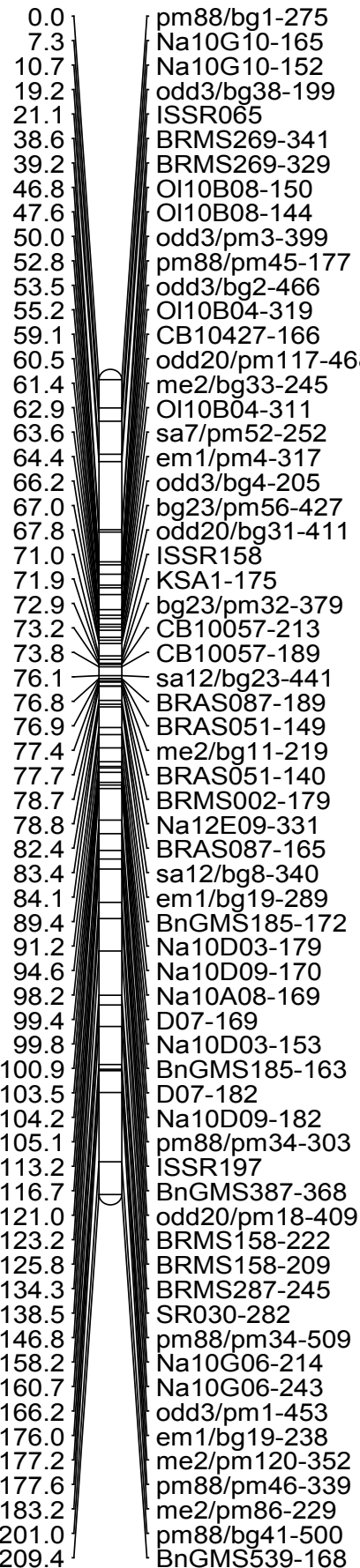

N15

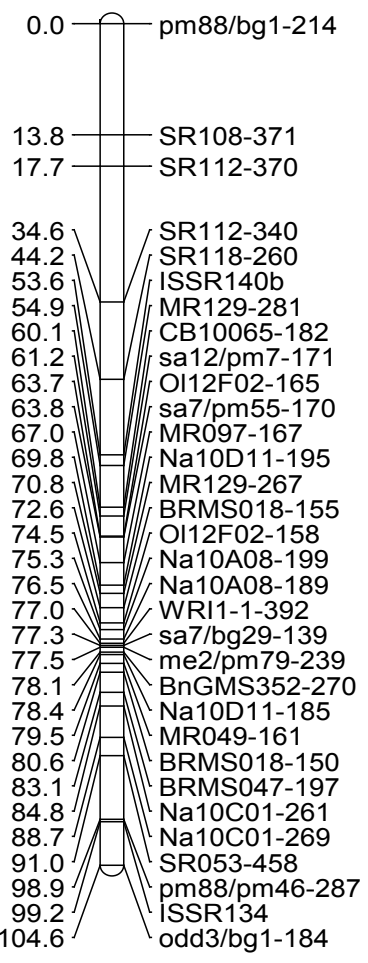

N18

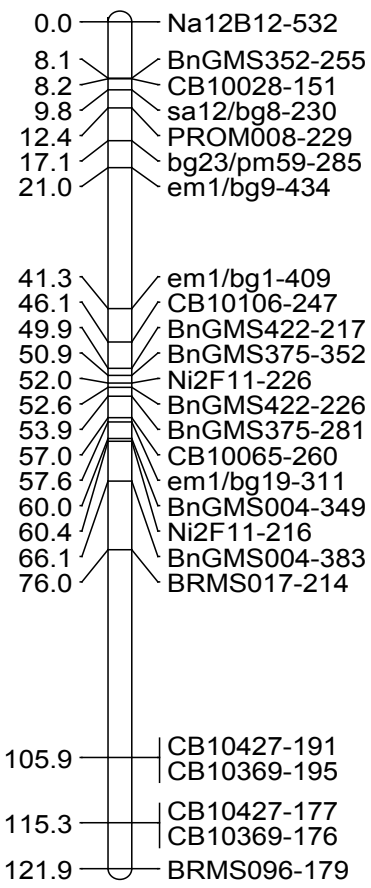

N16

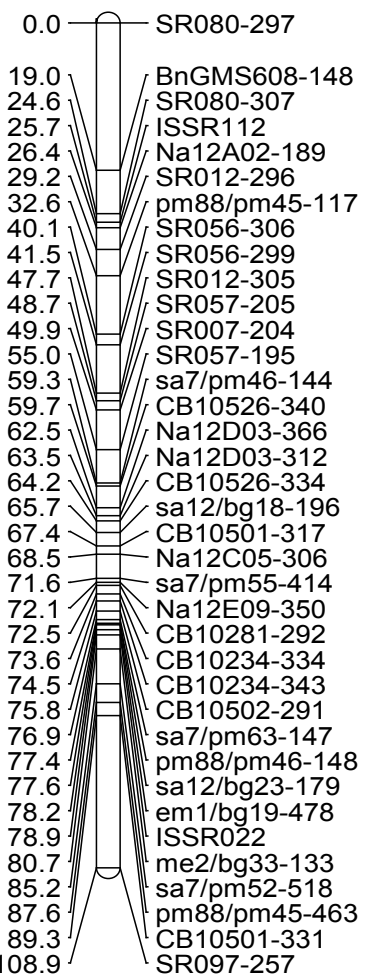

N19

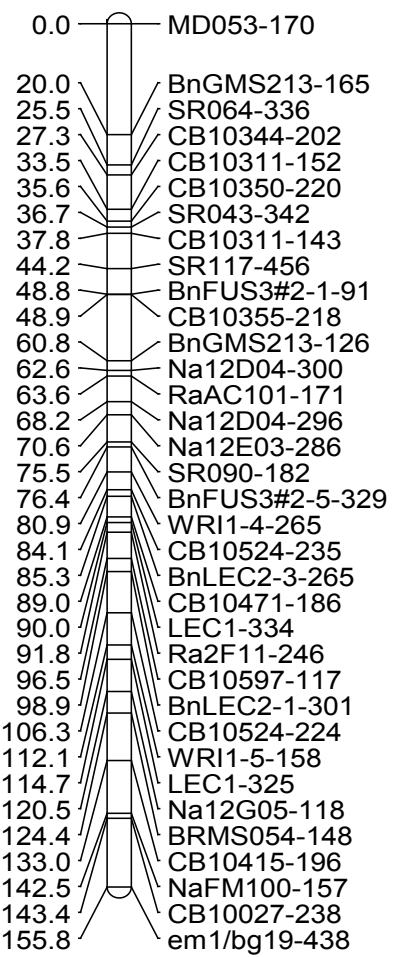

N17

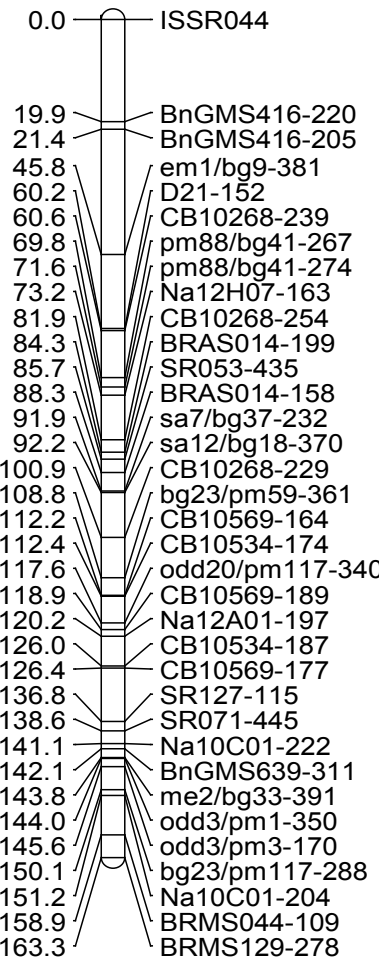

163.3 $\quad$ BRMS129-278 
Citation: Geng J, Javed N, McVetty PBE, Li G, Tahir M (2011) An Integrated Genetic Map for Brassica napus Derived from Double Haploid and Recombinant Inbred Populations. Hereditary Genetics 1:103. doi:10.4172/2161-1041.1000103

Page 13 of 16

for N14 and N01, respectively (Table 3).

\section{Comparison of individual maps and integrated genetic map}

Common markers among homologous LGs allowed the comparison of marker order between these individual maps and the integrated map. One hundred and ninety-four molecular markers were found in common between two populations; however, just 183 (94.3\%) were arranged onto the resulting 19 integrated LGs. Of these mapped common markers, 170 (92.9\%) markers were assembled onto the same LGs of the DH and RIL maps, nine located onto N01 (A genome) or N11 (C genome), N03 (A genome) or N13 (C genome), N04 (A genome) or N 14 (C genome), N07 (A genome) or N17 (C genome), N08 (A genome) or N18 (C genome) and N09 (A genome) or N19 (C genome) in the DH and RI maps, which suggests a synteny between the $\mathrm{A}$ and $\mathrm{C}$ genomes, while the remaining four markers distributed on different LGs whithout any relationship. The integrated map is generally in agreement with the two individual maps, and the two individual maps complement each other on the integrated map with small translocations. Regarding the length of LGs, five LGs (N02, $\mathrm{N} 03, \mathrm{~N} 07, \mathrm{~N} 18, \mathrm{~N} 19)$ in the integrated map are $10 \mathrm{cM}$ longer than in the individual maps, whereas eleven LGs (N01, N04, N05, N06, N08, N09, N10, N11, N13, N15, N17) in the integrated map are similar to the longer ones of the individual maps, while the remaining 3 LGs (N12, N14 and N16) were over $10 \mathrm{cM}$ shorter than the longer ones of

\section{INT01}

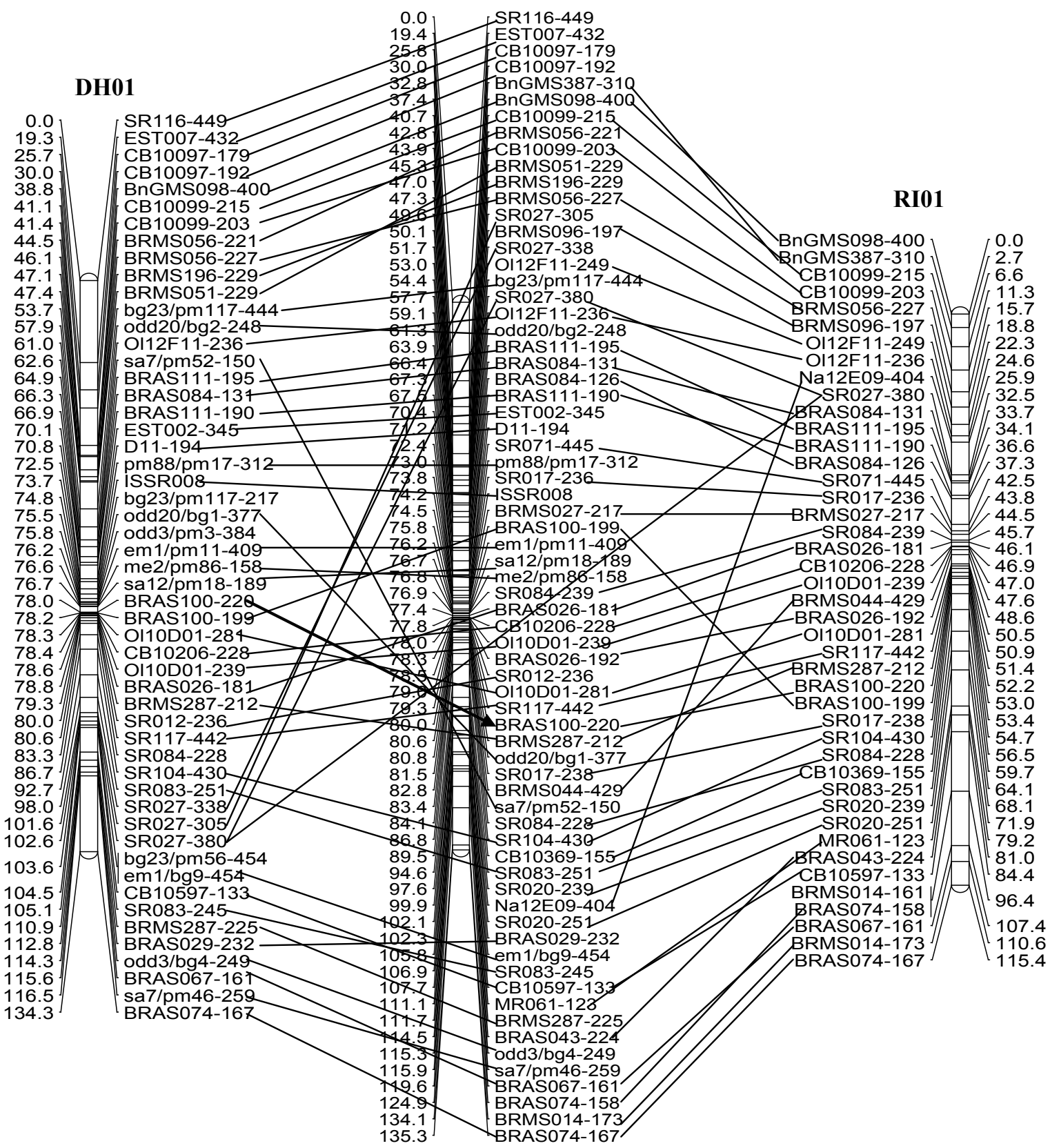

Note: DH01 represents LG01 in the DH-based map, INT01 represents LG01 in the integrated map, RI01 represents LG01 in the RIL-based map

Figure 4: Comparison of LG01 between DH-based map, RIL-based map and integrated map. 
the individual maps. This result revealed that most of LGs $(84.2 \%)$ in the integrated map were longer or similar to the related longer LG in individual maps, which resulted in a significantly longer coverage for the integrated map.

Regarding marker order, for example on LG 01 (Figure 4), most of the markers on individual maps shared the similar order; however, there were five translocatios (sa7/pm52-150, odd20/bg1-377, SR027-238, SR027-305 and SR027-338) detected between the DH and integrated maps. Similarly, between the RI and integrated maps, three markers (BRAS100-199, BRMS044-429 and Na12E09-404) translocated, however, only one translocation (SR027-380) was found between the $\mathrm{DH}$ and RI polulations.

\section{Discussion}

Generating sharable and lab-to-lab reproducibile results is becomming the most important and final purpose of genetic map construction, inspite of using different materials and experimental systems in different labs. In this study, two individual genetic maps were constructed with different types of markers, such as SSR, SRAP, ISSR and SCAR. Further these individual maps were combined into an integrated genetic map using DH and RI populations in Brassica napus. With the public SSR markers $(16 ; 34)$ found between individual maps, all the LGs were anchored to the corresponding chromosomes of Brassica napus. A total of 796 loci could be mapped onto the integrated map, whereas, only 620 and 349 markers were assembled on the DH and RI map, respectively. The integrated map covered a total genetic distance of $2464.9 \mathrm{cM}$, which is in the mean confidenceinterval estimates of genome length estimated as 2,127-2,480 cM (30), and thus, seemed to indicate near-complete genome coverage. The differences of map lengths in different studies are usually attribuable to scoring errors, type of markers, population size, recombination frenquency, LOD values, and the software employed [43]. Previously, Qi et al.[44], reported that the length and observed genome coverage in barley was greater with MAPMAKER than with JOINMAP. Pradhan et al. [45], also observed reduction in the total genetic length although they mapped more markers in comparision to other maps in Brassica juncea. In our study, similar evidence was observed that the length of the DH-based map was longer than the RI-based map due to one additional type of marker (SRAP) used, which filled large existing gaps on LGs, and eventually improved the genome coverage, assuming different markers are developed from different principles and amplifiy different genome regions, for example, SSR, ISSR and SRAP are based on SSR regions, inter-SSR regions and ORF regions, respectively.

Marker segregation distortion is a common phenomenon in crops $[27,46,47]$, especially in maps derived from $\mathrm{DH}$ population regardless of marker types [16,23,24,29]. This distortion probably results from gametic or zygotic selection, or from a specific selection derived from the production of plants in vitro microspore culture. In the study by Lombard and Delourme [30], the segregation bias was towards certain parents, with a corresponding region for microsporeculture responsiveness being identified in these parents. This finding suggests that distortion segregation is related to the genes controlling microspore responsiveness during haploid production. In our study, the result of goodness fit test ( $\chi^{2}$ test) revealed that $362(50.6 \%)$ and 174 (32.6\%) molecular markers violated the expected Mendelian segregation ratio of 1:1 for the $\mathrm{DH}$ and RI populations, repectively, the results suggested that the RI population is more nomal than the $\mathrm{DH}$ population. However, when a $\chi^{2}$ test was used on distorted markers for the $\mathrm{DH}$ population, $48.3 \%$ markers biased to Polo, and $51.7 \%$ markers biased to Topas, which suggests that this $\mathrm{DH}$ population is not distorted but rather a standard normal population. This conclusion is further supported by the similar response to microspore culture observed in both parents (data not published). Therefore, double $\chi^{2}$ tests are recommended for fitting Mendelian segregation ratio of 1:1. However, among the distorted markers of the RI population, $64.9 \%$ markers biased to Polo, and $35.1 \%$ markers biased to Topas, the result of second $\chi^{2}$ test showed a significant violation to the Mendelian segregation ratio of $1: 1$, this could be due to the smaller population size.

Genetic maps based on multiple populations and multiple types of molecular markers offer many advantages over a map based on a single population and one type of molecular marker. Likewise, in this study, a $\mathrm{DH}$ and a RI population were developed from the same $\mathrm{F}_{1}$ cross, which provided an opportunity to compare these populations as well as to contruct an integrated map from them. A higher percentage of markers (85.6\%) were assembled onto the main 19 LGs in the DH map than in the RI map (65.5\%). It could be argured that additional type of marker (SRAP) used in the $\mathrm{DH}$ population and the $\mathrm{DH}$ population size was larger than RI population. Most common markers (94.3\%) were mapped onto the same LGs and found at similar positions in individual maps, which suggests that DH and RI populations are both ideal for map constructions and complement each other.

Synteny is the preserved order of genes on chromosomes of related species which results from descent from a common ancestor. A chromosomal region of one species is said to be syntenic with a chromosomal region in another species if the regions carry two or more homologous genes. During evolution, chromosome rearrangements result in disruptions of synteny [30]. In our study, seventeen molecular markers showed synteny between the A genome and the $\mathrm{C}$ genome in $\mathrm{DH}$, eight markers in RI and 26 markers in the integrated map.

Compared with other crops such as rice and soybean [48], there are relatively few public SSR markers available in Brassica, although several research groups have presented a number of SSR markers with different technology $[16,34,35,37,49]$. SSR development has been improved since the early expensive technology of prob hybridization to an easy online source-based method. In our study, a large number of SSR sequences (including (AT)n, (CT)n, and (GA)n) were downloaded from a website, and a number of SSR primers were developed, tested and adopted to construct the genetic map. For the newly developed and linked SSR markers, 92 markers were assigned onto the A genome and 45 markes were mapped onto the $\mathrm{C}$ genome, nine distributed both on the $\mathrm{A}$ and $\mathrm{C}$ genome LGs. We believe that this method is accessible and efficient for SSR development and map construction. As SSR based markers, ISSRs are semi-arbitrary markers and are easy and quick to develop and use. Two kinds of ISSR primer, with or without an anchoring end were used in this study. Seventy-seven sucessfully amplified, 36 primers detected polymorphism between the two parents, and 29 ISSR markers were integrated onto the DH map, distributed on 13 LGs. All the mapped ISSR markers filled gaps, 3 of the markers, ISSR185, ISSR060 and ISSR44, were mapped onto the ends of N03, N04 and N17 respectively, which consquently improved map length by up to $43.4 \mathrm{cM}$, directly. In the RI population, 7 ISSR markers were mapped onto the ends of N04, N05, N11, N14, N15 and N16, respectively, which improved the map length by up to $81.5 \mathrm{cM}$, directly. This result indicates that ISSR is desirable and suitable for map construction in combination with 
Citation: Geng J, Javed N, McVetty PBE, Li G, Tahir M (2011) An Integrated Genetic Map for Brassica napus Derived from Double Haploid and Recombinant Inbred Populations. Hereditary Genetics 1:103. doi:10.4172/2161-1041.1000103

SSR markers. Sequence-related amplified polymorphisms (SRAP) have proved to be a simple approach and an efficient system for the framework contruction of genetic maps $[9,10]$. We selected 64 primer pairs for map construction, and 199 polymorphic bands were detected from these primer pairs, with 191 of them assigned onto the DH map, distributed on 19 LGs, which saturated the map greatly. This result supported that SRAP could be valuable to saturate the genetic map.

Nineteen SCAR markers related to fatty acid synthsis pathway genes were integrated onto the present maps. This result offers detailed references for related agronomic quantitative trait loci (QTL) mapping and marker-assisted selection (MAS). The current maps and previously developed genetic maps could play an important role in QTL mapping and map-based gene coloning in Brassica napus.

\section{Acknowledgements}

This work was supported by the Natural Sciences and Engineering Research Council (NSERC) of Canada and industrial and government partners, through the Green Crop Networks (GCN) Research Network Canada.

\section{References}

1. Paterson AH (1996) Making genetic maps In AH Paterson (ed) Genome mapping in plants. RG Landes Company San Diego California USA pp 23-39.

2. Maheswaran M (2004) Molecular Markers: History Features and Applications. Advanced Biotech August 2004

3. Gupta PK, Varshney RK (2000) The development and use of microsatellite markers for genetic analysis and plant breeding with emphasis on bread wheat. Euphytica 113: 163-185.

4. Studer B, Kölliker R, Muylle H, Asp T, Frei U, et al. (2010) EST-derived SSR markers used as anchor loci for the construction of a consensus linkage map in ryegrass (Lolium spp). BMC Plant Biology 10: 177.

5. Künzel G, Waugh R (2002) Integration of microsatellite markers into the translocation-based physical RFLP map of barley chromosome $3 \mathrm{H}$. Theor Appl Genet 105: 660-665.

6. Xu Z, Kohel RJ, Song G, Cho J, Yu J, et al. (2008) An integrated genetic and physical map of homoeologous chromosomes 12 and 26 in Upland cotton (G. hirsutum L). BMC Genomics 9: 108.

7. Li X, Ramchiary N, Choi SR, Nguyen DV, Hossain MJ, et al. (2010) Development of a high density integrated reference genetic linkage map for the multinational Brassica rapa Genome Sequencing Project. Genome 53: 939-947.

8. González VM, Garcia-Mas J, Arús P, Puigdomènech P (2010) Generation of a BAC-based physical map of the melon genome. BMC Genomics 11: 339.

9. Li G, Quiros CF (2001) Sequence-related amplified polymorphism (SRAP) a new marker system based on a simple PCR reaction: its application to mapping and gene tagging in Brassica. Theor Appl Genet 103: 455-461.

10. Sun Z, Wang Z, Tu J, Zhang J, Yu F (2007) An ultradense genetic recombination map for Brassica napus consisting of 13551 SRAP markers. Theor Appl Genet 114: $1305-1317$

11. Zietkiewicz E, Rafalski A, Labuda D (1994) Genome fingerprinting by simple sequence repeat (SSR)-anchored polymerase chain reaction amplification. Genomics 20: 176-183.

12. Flandez-Galvez H, Ford R, Pang ECK, Taylor PWJ ( 2003) An intraspecific linkage map of the chickpea (Cicer arietinum $L$ ) genome based on sequence tagged microsatellite site and resistance gene analog markers. Theor Appl Genet 106: 1447-1456.

13. Hashizume T, Shimamoto I, Hirai M (2003) Construction of a linkage map and QTL analysis of horticultural traits for watermelon [Citrullus lanatus (THUNB) MATSUM \& NAKAI] using RAPD, RFLP and ISSR markers. Theor Appl Genet 106: 779-785.

14. Rubeena RF, Taylor PWJ (2003) Construction of an intraspecific linkage map of lentil (Lens culinaris ssp culinaris).Theor Appl Genet 107: 910-916.

15. Gupta SK, Souframanien J, Gopalakrishna T (2008) Construction of a genetic linkage map of black gram Vigna mungo (L) Heppe based on molecular markers and comparative studies. Genome 5: 628-637.

16. Piquemal J, Cinquin E, Couton F, Rondeau C, Seignoret E, et al. (2005) Construction of an oilseed rape (Brassica napus L.) genetic map with SSR markers. Theor Appl Genet 111: 1514-1523.

17. Mace ES, Rami JF, Bouchet S, Klein PE, Klein RR, et al. (2009) A consensus genetic map of sorghum that integrates multiple component maps and highthroughput Diversity Array Technology (DArT) markers. BMC Plant Biology 9 13.

18. Isobe S, Kölliker R, Hisano H, Sasamoto S, Wada T, et al. (2009) Construction of a consensus linkage map for red clover (Trifolium pratense L). BMC Plant Biology 9: 57.

19. Lee HR, Bae1 IH, Park SW, Kim HJ, Min WK, et al. (2009) Construction of an integrated pepper map using RFLP SSR CAPS AFLP WRKY rRAMP and BAC end sequences. Mol Cells 27: 21-37.

20. Hwang TY, Sayama1 T, Takahashi M, Takada Y, Nakamoto Y, et al. (2009) High-density integrated linkage map based on SSR markers in soybean. DNA Research 16: 213-225.

21. Córdoba JM, Chavarro C, Schlueter JA, Jackson SA, Blair MW (2010) Integration of physical and genetic maps of common bean through BACderived microsatellite markers. BMC Genomics 11: 436.

22. Basunanda P, Radoev M, Ecke W, Friedt W, Becker HC, et al. (2010) Comparative mapping of quantitative trait loci involved in heterosis for seedling and yield traits in oilseed rape (Brassica napus L). Theor Appl Genet 120: 271 281.

23. Landry BS, Hubert N, Etoh T, Harada JJ, Lincoln SE (1991) A genetic map for Brassica napus based on restriction fragment length polymorphisms detected with expressed DNA sequences. Genome 34: 543-552.

24. Ferreira ME, Williams PH, Osborn TC (1994) RFLP mapping of Brassica napus using doubled haploid lines. Theor Appl Genet 89: 615-621.

25. Sharpe AG, Parkin IAP, Keith DJ, Lydiate DJ (1995) Frequent nonreciprocal translocations in the amphidiploid genome of oilseed rape (Brassica napus). Genome 38: 1112-1121.

26. Uzunova M, Ecke W, Weissleder K, Robbelen G (1995) Mapping the genome of rapeseed (Brassica napus L) I. Construction of an RFLP linkage map and localization of QTLs for seed glucosinolate content. Theor Appl Genet 90: 194 204

27. Foisset N, Delmourme R, Barret P, Hubert N, Landry BS, et al. (1996) Molecular mapping analysis in Brassica napus using isozyme RAPD and RFLP markers on a doubled haploid progeny. Theor Appl Genet 93: 1017-1025.

28. Cheung WY, Friesen L, Rakow GFW, Se'guin-Swartz G, Landry BS (1997) A RFLP-based linkage map of mustard [Brassica juncea (L) Czern and Coss] Theor Appl Genet 94: 841-851.

29. Chen G, Geng J, Rahman M, Liu X, Tu J, et al. (2010) Identification of QTL for oil content seed yield and floweing time in oilseed rape (Brassica napus) Euphytica 175: 161-174.

30. Lom Mccouch bard V, Delourme R (2001) A consensus linkage map for rapeseed (Brassica napus $L$ ) construction and integration of three individual maps from DH populations. Theor Appl Genet 103: 491-507.

31. Udall JA, Quijada PA, Osborn TC (2005) Detection of chromosomal rearrangements derived from homoeologoous recombination in four mapping population of Brassica napus L. Genetics 169: 967-979.

32. Delourme R, Falentin C, Huteau V, Clouet V, Horvais R, et al. (2006) Genetic control of oil content in rapeseed (Brassica napus L). Theor Appl Genet 113 1331-1345.

33. Suwabe K, Morgan C, Bancroft lan (2008) Integration of brassica A genome genetic linkage map between Brassica napus and Brassica rapa. Genome 51 169-176.

34. Cheng X, Xu J, Xia S, Gu J, Yang Y, et al. (2009) Development and genetic mapping of microsatellite markers from genome survey sequences in Brassica napus. Theor Appl Genet 118: 1121-1131.

35. Xu J, Qian X, Wang X, Li R, Cheng X, et al. (2010) Construction of an integrated 
Citation: Geng J, Javed N, McVetty PBE, Li G, Tahir M (2011) An Integrated Genetic Map for Brassica napus Derived from Double Haploid and Recombinant Inbred Populations. Hereditary Genetics 1:103. doi:10.4172/2161-1041.1000103

Page 16 of 16

genetic linkage map for the A genome of Brassica napus using SSR markers derived from sequenced BACs in Brassica rapa. BMC Genomics 2010 11: 594.

36. Lowe AJ, Moule C, Trick M, Edwards KJ (2004) Efficient large-scale development of microsatellites for marker and mapping applications in Brassica crop species. Theor Appl Genet 108: 1103-1112.

37. Geng J, Zhu C, Zhang X, Cheng Y, Zhang Y, et al. (2007) A genetic linkage map of nonheading Chinese cabbage. J Amer Soc Hort Sci 132: 816-823.

38. Van Ooijen JW, Voorrips RE (2001) Join Map version 3.0: software for the calculation of genetic linkage maps Plant research international Wageningen, the Netherlands.

39. Kosambi DD (1943) The estimation of map distances from recombination values. Ann Eugen 12: 172-175.

40. Gosselin I, Zhou Y, Bousquet J, Isabel N (2002) Megagametophte-derivered linkage maps of white spruce (Picea glaucea) based on RAPD SCAR and ESTP markers. Theor Appl Genet 104: 987-997.

41. Qi X, Stam P, Lindhout $P$ (1996) Comparison and integration of four barley genetic maps. Genome 39: 379-394.

42. Pradhan AK, Gupta V, Mukhopadhyay A, Arumugam N, Sodhi YS, et al. (2003) A high-density linkage map in Brassica juncea (Indian mustard) using AFLP and RFLP markers. Theor Appl Genet 106: 607-614.

43. Voorrips RE, Jongerius MC, Kanne HJ (1997) Mapping of two genes for resistance to clubroot (Plasmodiophora brassicae) in a population of doubled haploid lines of Brassica oleracea by means of RFLP and AFLP markers. Theor Appl Genet 94: 75-82.

44. Kim JH, Jang CS, Cho KW, Lim YP (1999) AFLP and RAPD mapping of chinese cabbage (Brassica rapa L. var pekinensis) In: Proceedings of the plant and animal genome conference VII San Diego California USA 17-21.

45. Mc Couch SR, Teytelman L, Xu YB, Lobos KB, Clare K, et al. (2002) Development and mapping of 2240 new SSR markers for rice (Oryza sativa L). DNA Res 9: 199-207.

46. Suwabe K, Iketani H, Nunome T, Kage T, Hirai M (2002) Isolation and characterization of microsatellites in Brassica rapa L. Theor Appl Genet 104: 1092-1098.

47. Hu Y, Wu G, Gao Y, Wu Y, Xiao L, et al. (2009) Breeding response of transcrip profiling in developing seeds of Brassica napus. BMC Molecular Biology 10: 49.

48. Suwabe K, Tsukazaki H, Iketani H, Hatakeyama K, Kondo M, et al. (2006) Simple sequence repeat-based comparative genomics between Brassica rapa and Arabidopsis thaliana: The genetic origin of clubroot resistance. Genetics 173: 309-319

49. Truco M J, Antonise R, Lavelle D, Ochoa O, Kozik A, et al. (2007) A highdensity integrated genetic linkage map of lettuce (Lactuca spp). Theor Appl Genet 115: 735-746.
This article was originally published in a special issue, Metabolic Syndrome handled by Editor(s). Dr. Agathocles Tsatsoulis, University Hospital of loannina, USA; Dr. Christa Buechler, University Hospital Regensburg, USA
Submit your next manuscript and get advantages of OMICS Group submissions

Unique features:

- User friendly/feasible website-translation of your paper to 50 world's leading languages

Audio Version of published paper

Digital articles to share and explore

Special features:

- 200 Open Access Journals

- 15,000 editorial team

21 days rapid review process

Quality and quick editorial, review and publication processing

- Indexing at PubMed (partial), Scopus, DOAJ, EBSCO, Index Copernicus and Google Scholar etc

- Sharing Option: Social Networking Enabled

Authors, Reviewers and Editors rewarded with online Scientific Credits

Better discount for your subsequent articles

Submit your manuscript at: http://www.omicsonline.org/submission/ 